\title{
Stability and Completion of Zeno Equilibria in Lagrangian Hybrid Systems
}

\author{
Yizhar Or and Aaron D. Ames, Associate Member, IEEE
}

\begin{abstract}
This paper studies Lagrangian hybrid systems, which are a special class of hybrid systems modeling mechanical systems with unilateral constraints that are undergoing impacts. This class of systems naturally display Zeno behavior-an infinite number of discrete transitions that occur in finite time, leading to the convergence of solutions to limit sets called Zeno equilibria. This paper derives simple conditions for stability of Zeno equilibria. Utilizing these results and the constructive techniques used to prove them, the paper introduces the notion of a completed hybrid system which is an extended hybrid system model allowing for the extension of solutions beyond Zeno points. A procedure for practical simulation of completed hybrid systems is outlined, and conditions guaranteeing upper bounds on the incurred numerical error are derived. Finally, we discuss an application of these results to the stability of unilaterally constrained motion of mechanical systems under perturbations that violate the constraint.
\end{abstract}

Index Terms-Langrangian hybrid systems, stability, unilateral contact constraints, Zeno behavior.

\section{INTRODUCTION}

$\mathbf{H}$ YBRID dynamical systems are systems that display both continuous and discrete behavior [17], [27], [44]. Systems of this form are used to model a myriad of applications, ranging from biological systems to chemical processes to robotics. A fundamental phenomenon which is unique to hybrid system is Zeno behavior - an infinite number of discrete transitions that occur in a finite amount of time, called the Zeno time. Points to which Zeno solutions converge are called Zeno equilibria, which are fixed points of the discrete dynamics but not the continuous dynamics. Despite the simplicity of these definitions, understanding Zeno behavior on a fundamental level presents difficult and intriguing problems: Can simple conditions for the existence of Zeno behavior be obtained? How does the existence of Zeno behavior relate to the convergence properties, or stability, of hybrid systems? What happens to the solution of the system after the Zeno time?

In this paper, we address these fundamental questions by focusing on a special class of hybrid systems termed Lagrangian hybrid systems, which model mechanical systems undergoing

Manuscript received December 30, 2008; revised September 03, 2009; accepted August 28, 2010. Date of publication September 27, 2010; date of current version June 08, 2011. This work was supported by a Fulbright Postdoctoral Fellowship and Bikura Scholarship of the Israeli Science Foundation. Recommended by Associate Editor H. Ishii.

Y. Or is with the Faculty of Mechanical Engineering, Technion-Israel Institute of Technology, Haifa 32000, Israel (e-mail: izi@tx.technion.ac.il).

A. D. Ames is with the Mechanical Engineering Department, Texas A\&M University, College Station, TX 77843 USA (e-mail: aames@tamu.edu).

Color versions of one or more of the figures in this paper are available online at http://ieeexplore.ieee.org.

Digital Object Identifier 10.1109/TAC.2010.2080790 impacts. In particular, we consider a configuration space, a Lagrangian modeling a mechanical system, and a unilateral constraint function that gives the set of admissible configurations for this system. This class of systems were widely studied in the literature [7], [25], [36], and are traditionally modeled as hybrid systems [17], [29], [31]. Hybrid systems of this form naturally display Zeno behavior, and therefore provide the ideal class of systems in which to gain an intuitive understanding of this phenomenon, which has physically meaningful interpretations.

\section{A. Literature Review}

1) Zeno Behavior: Due to the subtle and complex nature of Zeno behavior, it has been studied in many forms and from many different perspectives. Most of the conditions for existence of Zeno behavior are necessary and tend to be very conservative; see [49], [50] for general hybrid systems, and [9], [41] for linear complementarity systems. Until recently, sufficient conditions for Zeno behavior were more rare [1]. Necessary and sufficient conditions for Zeno behavior in a particular class of controlled hybrid systems were found in [18]. In the context of Lagrangian hybrid systems, Lamperski and Ames [21] provided Lyapunov-like conditions for Zeno behavior near isolated Zeno equilibrium points. Ames et al. [2] derived sufficient conditions for Zeno behavior in a special class of hybrid systems, called first quadrant hybrid systems. This idea was then generalized in [22] to set-valued first quadrant hybrid systems, with application to nonisolated Zeno equilibria in Lagrangian hybrid systems.

2) Stability in Zeno Hybrid Systems: In dynamical systems theory, a classical definition of stability of an equilibrium point is due to Lyapunov [26]. This definition states that an equilibrium point $x_{0}$ is stable if there exists a small open neighborhood of initial conditions near $x_{0}$, under which the solution stays bounded within an arbitrarily small neighborhood of $x_{0}$. This notion naturally generalizes to hybrid systems, e.g., [17], [47], and, in particular, to Zeno solutions [39], [42]. Only recently, Goebel and Teel [15] defined the notion of uniform Zeno stability, which captures the fact that the finite convergence (Zeno) time can also be bounded in terms of the neighborhood of initial conditions.

3) Completing Hybrid Systems Solutions Beyond Zeno Point: The problem of carrying solutions of hybrid systems beyond Zeno times is even more involved. It is often argued that Zeno behavior represents an inherent limitation of the hybrid system model, where the solution reaches a point at which the model is no longer valid, and cannot predict the true behavior of the physical system. On a more practical level, in numerical simulations of hybrid systems, Zeno solutions lead to problems of computational inefficiency and numerical errors [20]. Some attempts to 
shed light on the behavior of the solution past the Zeno point are as follows. In the closely related class of switched systems [23], Zeno solutions often occur, associated with finite-time convergence to a switching surface in state space, along with increasingly fast switching events near this surface, called chattering. In that case, the solutions can be extended by considering the set-valued Filippov solution [13], which involves sliding along the switching surface. Another technique that has been proposed in the hybrid systems literature is that of regularization, which was illustrated for particular examples in [20]. This technique is based on perturbing the dynamical system in order to obtain non-Zeno solution, and then taking the limit as the perturbation goes to zero. A more formal procedure for obtaining generalized solutions of Zeno hybrid system via regularization was presented in [14], [38]. Following similar guidelines, generalized solutions for unilaterally constrained mechanical systems are analyzed in [29].

In the particular class of Lagrangian hybrid systems, the special structure of the system clearly indicates what the solution "should" do after convergence to a Zeno point. Ames et al. [5] have made the key observation that Zeno limit points lie on the zero-level set of the unilateral constraint function, with their velocity vector tangent to this constraint surface. Therefore, they postulated that after the Zeno time, the system switches to holonomically constrained dynamics, where the solution slides along the zero-level set of the constraint function. However, the authors in [5] overlooked the fact that there should also be a transition from the constrained motion back to the hybrid dynamics, which is associated with solutions leaving the constraint surface.

\section{B. Summary of Contributions}

We now summarize the key contributions of this paper. First, focusing on Lagrangian hybrid systems, in Section III we formally define the type of stability that Zeno equilibria in such systems can display: bounded-time local stability, which is similar in spirit to the uniform Zeno stability defined in [15]. We derive necessary and sufficient conditions for the stability of Zeno equilibria in Lagrangian hybrid systems, and show that the stability conditions are exactly the same as the sufficient conditions for existence of Zeno behavior presented in [22]. Moreover, our stability proof is constructive, in the sense that it derives explicit bounds on the neighborhood of initial conditions that guarantee convergence of the solution in an arbitrarily small Zeno time while staying bounded within any given neighborhood.

In Section IV, we continue the work of [5] and propose a formal method for "completing" solutions of Lagrangian hybrid systems beyond Zeno points. This is done by defining a completed hybrid system, consisting of a domain with hybrid dynamics and a domain with constrained dynamics, together with transitions between them. The transition from the hybrid dynamics to constrained motion occurs after a Zeno solution converges to a Zeno equilibrium point. The transition back from constrained motion to the hybrid dynamics occurs when the unilateral constraint force vanishes.

While these new notions are theoretically valuable, they are not practically useful, since in general, the Zeno point cannot be computed exactly, as it requires computation of infinite number of discrete transitions. Any practical simulation essentially involves truncation of the Zeno solution after a finite number of transitions, leading to unavoidable numerical errors. Therefore, in Section V we present conditions for reliable truncation, which guarantee that the exact solution actually exhibits a true Zeno behavior, and that the numerical error incurred by the finite truncation is less than a pre-specified bound. The derivation of these conditions is done by exploiting the constructive stability proof in Section III, which derives explicit bounds on the deviation of the solution from the exact (unknown) Zeno point.

Finally, in Section VII we discuss a key interpretation of the results to stability of unilaterally constrained mechanical systems under perturbations that violate the constraint.

\section{Significance}

The physical significance of Zeno behavior in mechanical systems is that it corresponds to a sequence of impacts which eventually converges to contact re-establishment, as in the examples of a bouncing ball or tossing a dice. In reality, the precise dynamics of such systems during the short periods of impact involves complicated interactions that can be analyzed using more refined models of contact mechanics. In order to bypass the difficulties involved in these models, those interactions are lumped into rigid-body impacts, which are instantaneous events of discontinuous velocity jumps. Therefore, in some sense, Zeno equilibria and Zeno behavior in general are artifacts of this simplifying model. Yet by analyzing the stability of Zeno equilibria, the claim is that one is analyzing the stability of the equilibrium point that would exist if the more detailed contact dynamics were considered. Moreover, the completion process that will be introduced in this paper allows one to continue time forward in the same way it would if more complex models of the systems were considered. Thus, our study provides insight into the qualitative behavior of the physical system while simultaneously reaping the benefit of considering a simpler model of the dynamics as a hybrid system.

Our work can be utilized as a theoretical framework for analysis and simulation of mechanical or robotic control systems involving intermittent contacts. Such systems were previously studied as control problems in the literature, e.g., [7], [12], [35], [46]. However, these works did not explicitly address the subtle issues related to simulating the Zeno behavior of the system. Since Zeno behavior can occur in a large class of systems for which it is desirable to control and analyze, this work has the potential to impact a wide variety of applications.

The importance of understanding Zeno behavior in hybrid systems and its relationship to control can best be seen by considering a specific example: bipedal robotic walking. Bipeds are naturally modeled as hybrid (control) systems [4], [16], [28], [46]: when the leg is swinging, its motion is governed by (controlled, underactuated) Euler-Lagrange equations, and when the foot strikes the ground the dynamics are discrete due to the instantaneous impact event. For bipedal robots, feedback control laws can be developed that result in stable walking, i.e., stable hybrid periodic orbits [3], [43], [46]. In obtaining a hybrid model of a bipedal robot, it is traditionally assumed that impacts are perfectly plastic, resulting in instantaneous switch to a sticking contact. In reality, this assumption may be difficult 
to guarantee and in the case when it is violated, the end result is Zeno behavior in the hybrid model. Therefore, understanding the stability of Zeno equilibria and how to complete hybrid systems will be essential in understanding how these system behave in reality. To provide a specific example, the results of this paper have been recently used to guarantee the existence of a limit cycle in a system with non-plastic impacts given the existence of stable limit cycles under plastic impacts [31]. In the context of bipedal walking, results of this form ensure that the walking gait is robust with respect to changes in the impact model.

\section{BASIC TERMINOLOGY}

In this section we introduce the terminology for the paper and define the Lagrangian hybrid system. Then we introduce the Zeno behavior and define Zeno equilibria.

\section{A. Lagrangian Hybrid Systems}

We now introduce the notion of a hybrid Lagrangian and the associated Lagrangian hybrid system. First, we review the notion of a simple hybrid system.

Definition 1: A simple hybrid system is a tuple $\mathrm{H}=$ $(D, G, R, f)$, where :

- $D$ is a smooth manifold called the domain;

- $G$ is an embedded submanifold of $D$ called the guard;

- $R$ is a smooth map $R: G \rightarrow D$ called the reset map;

- $f$ is a vector field on the manifold $D$.

This paper focuses on simple hybrid systems, having a single domain, guard and reset map. A general hybrid system (see [27]), which is not discussed here, consists of a collection of domains, guards, reset maps and vector fields as indexed by an oriented graph.

1) Hybrid Executions: An execution of a simple hybrid system $\mathrm{H}$ is a tuple $\chi^{\mathrm{H}}=(\Lambda, \mathcal{I}, \mathcal{C})$, where:

- $\Lambda=\{0,1,2, \ldots\} \subseteq \mathbb{N}$ is an indexing set;

- $\mathcal{I}=\left\{I_{i}\right\}_{i \in \Lambda}$ is a hybrid interval where $I_{i}=\left[\tau_{i}, \tau_{i+1}\right]$ if $i, i+1 \in \Lambda$ and $I_{N-1}=\left[\tau_{N-1}, \tau_{N}\right]$ or $\left[\tau_{N-1}, \tau_{N}\right)$ or $\left[\tau_{N-1}, \infty\right)$ if $|\Lambda|=N, N$ finite. Here, $\tau_{i}, \tau_{i+1}, \tau_{N} \in \mathbb{R}$ and $\tau_{i} \leq \tau_{i+1}$

- $\mathcal{C}=\left\{c_{i}\right\}_{i \in \Lambda}$ is a collection of integral curves of $f$, i.e., $\dot{c}_{i}(t)=f\left(c_{i}(t)\right)$ for $t \in I_{i}, i \in \Lambda$,

The following conditions hold for every $i, i+1 \in \Lambda$ :

(i) $c_{i}\left(\tau_{i+1}\right) \in G$,

(ii) $\tau_{i+1}=\min \left\{t \in I_{i}: c_{i}(t) \in G\right\}$.

(iii) $c_{i+1}\left(\tau_{i+1}\right)=R\left(c_{i}\left(\tau_{i+1}\right)\right)$.

The initial condition for the execution is $c_{0}\left(\tau_{0}\right)$.

2) Lagrangians: Let $Q$ be the $n$-dimensional configuration space for a mechanical system (assumed to be a smooth manifold) and $T Q$ the tangent bundle of $Q$. In this paper, we will consider Lagrangians, $L: T Q \rightarrow \mathbb{R}$, describing mechanical, or robotic, systems, which are Lagrangians of the form

$$
L(q, \dot{q})=\frac{1}{2} \dot{q}^{T} M(q) \dot{q}-V(q)
$$

where $M(q)$ is the (positive definite) inertial matrix, $1 / 2 \dot{q}^{T} M(q) \dot{q}$ is the kinetic energy and $V(q)$ is the potential energy. In this case, the Euler-Lagrange equations yield the (unconstrained) equations of motion for the system

$$
M(q) \ddot{q}+C(q, \dot{q}) \dot{q}+N(q)=0
$$

where $C(q, \dot{q})$ is the Coriolis matrix (cf. [30]) and $N(q)=$ $\partial V / \partial q(q)$. Setting $x=(q, \dot{q})$, the Lagrangian vector field, $f_{L}$, associated to $L$ takes the familiar form

$$
\dot{x}=f_{L}(x)=\left(\begin{array}{c}
\dot{q} \\
M(q)^{-1}(-C(q, \dot{q}) \dot{q}-N(q))
\end{array}\right) .
$$

This process of associating a dynamical system to a Lagrangian will be mirrored in the setting of hybrid systems. First, we introduce the notion of a hybrid Lagrangian.

Definition 2: A simple hybrid Lagrangian is defined to be a tuple $\mathbf{L}=(Q, L, h)$, where:

- $Q$ is the configuration space;

- $L: T Q \rightarrow \mathbb{R}$ is a Lagrangian, assumed to be of the form (1);

- $h: Q \rightarrow \mathbb{R}$ provides a unilateral constraint on the configuration space $h(q) \geq 0$; we assume that the zero level set $h^{-1}(0)$ is a smooth manifold.

3) Simple Lagrangian Hybrid Systems: For a Lagrangian (1), there is an associated dynamical system (3). Similarly, given a hybrid Lagrangian $\mathbf{L}=(Q, L, h)$ the simple Lagrangian hybrid system (SLHS) associated to $\mathbf{L}$ is the simple hybrid system $\mathrm{H}_{\mathbf{L}}=\left(D_{\mathbf{L}}, G_{\mathbf{L}}, R_{\mathbf{L}}, f_{\mathbf{L}}\right)$. First, we define

$$
\begin{aligned}
D_{\mathbf{L}} & =\{(q, \dot{q}) \in T Q: h(q) \geq 0\}, \quad \text { and } \\
G_{\mathbf{L}} & =\{(q, \dot{q}) \in T Q: h(q)=0 \text { and } d h(q) \dot{q} \leq 0\} \\
& \text { where } \\
d h(q) & =\left(\frac{\partial h}{\partial q}(q)\right)^{T}=\left(\begin{array}{lll}
\frac{\partial h}{\partial q_{1}}(q) \quad \cdots \quad & \left.\frac{\partial h}{\partial q_{n}}(q)\right) .
\end{array}\right.
\end{aligned}
$$

In this paper, we adopt the reset map ([8]): $R_{\mathbf{L}}(q, \dot{q})=$ $\left(q, P_{\mathbf{L}}(q, \dot{q})\right)$, which is based on the impact law

$$
P_{\mathbf{L}}(q, \dot{q})=\dot{q}-(1+e) \frac{d h(q) \dot{q}}{d h(q) M(q)^{-1} d h(q)^{T}} M(q)^{-1} d h(q)^{T}
$$

where $0 \leq e \leq 1$ is the coefficient of restitution, which is a measure of the energy dissipated through impact. The impact law (4) corresponds to rigid-body collision under the assumption of frictionless impact. Examples of more complicated impact laws that account for friction can be found in [8], [10]. Finally, $f_{\mathbf{L}}=f_{L}$ is the Lagrangian vector field associated to $L$ in (3).

\section{B. Zeno Behavior and Zeno Equilibria}

We now introduce the notions of Zeno behavior and Zeno equilibria. More importantly, we review the sufficient conditions for Zeno behavior that will motivate the result of the next section, in that our sufficient conditions for the stability of Zeno equilibria utilize exactly the same conditions; that is, in Lagrangian hybrid systems, the existence of Zeno behavior and the stability of Zeno equilibria can be detected with the same simple and easily verifiable conditions. 
1) Zeno Behavior: An execution $\chi^{\mathrm{H}}$ is Zeno if $\Lambda=$ $\mathbb{N}$ and $\lim _{i \rightarrow \infty} \tau_{i}=\tau_{\infty}<\infty$. Here, $\tau_{\infty}$ is called the Zeno time. If $\chi^{\mathrm{H}_{\mathbf{L}}}$ is a Zeno execution of a Lagrangian hybrid system then its Zeno point is defined to be $x_{\infty}=\left(q_{\infty}, \dot{q}_{\infty}\right)=\lim _{i \rightarrow \infty} c_{i}\left(\tau_{i}\right)=\lim _{i \rightarrow \infty}\left(q_{i}\left(\tau_{i}\right), \dot{q}_{i}\left(\tau_{i}\right)\right)$. Zeno points are intricately related to a type of equilibrium points that are unique to hybrid systems: Zeno equilibria.

Definition 3: A Zeno equilibrium point of a simple hybrid system $\mathrm{H}$ is a point $x^{*} \in G$ such that $R\left(x^{*}\right)=x^{*}$ and $f\left(x^{*}\right) \neq 0$.

Note that, by definition, Zeno equilibria are fixed points of the discrete dynamics of a hybrid system but not fixed points of the continuous dynamics. If $\mathrm{H}_{\mathbf{L}}$ is a Lagrangian hybrid system, then due to the special form of these systems we find that a point $x^{*}=\left(q^{*}, \dot{q}^{*}\right)$ such that $f_{L}\left(x^{*}\right) \neq 0$ is a Zeno equilibrium point iff $\dot{q}^{*}=P_{\mathbf{L}}\left(q, \dot{q}^{*}\right)$, with $P_{\mathbf{L}}$ given in (4). In particular, the special form of $P_{\mathbf{L}}$ implies that this hold iff $d h\left(q^{*}\right) \dot{q}^{*}=0$. Therefore, the set of all Zeno equilibria for a Lagrangian hybrid system is given by the hypersurfaces in $G_{\mathbf{L}}$

$$
Z=\left\{(q, \dot{q}) \in G_{\mathbf{L}}: d h(q) \dot{q}=0 \quad \text { and } \quad f_{L}(x) \neq 0\right\} .
$$

Note that if $\operatorname{dim}(Q)>1$, the Zeno equilibria in Lagrangian hybrid systems are always non-isolated (see [21]) — this motivates the study of such equilibria.

2) Sufficient Conditions for Zeno Behavior: Let $\ddot{h}(q, \dot{q})$ be the acceleration of $h(q)$ along trajectories of the unconstrained dynamics (2), given by

$$
\ddot{h}(q, \dot{q})=\dot{q}^{T} H(q) \dot{q}-d h(q) M(q)^{-1}(C(q, \dot{q}) \dot{q}+N(q))
$$

where $H(q)$ is the Hessian of $h$ at $q$. The following theorem, which was proven in [22], provides sufficient conditions for existence of Zeno executions in the vicinity of a Zeno equilibrium point.

Theorem 1 ([22]): Let $\mathrm{H}_{\mathbf{L}}$ be a simple Lagrangian hybrid system and let $\left(q^{*}, \dot{q}^{*}\right)$ be a Zeno equilibrium point of $\mathrm{H}_{\mathbf{L}}$. Then if $e<1$ and $\ddot{h}\left(q^{*}, \dot{q}^{*}\right)<0$, there exists a neighborhood $W \subset D_{\mathbf{L}}$ of $\left(q^{*}, \dot{q}^{*}\right)$ such that for every $\left(q_{0}, \dot{q}_{0}\right) \in W$, there is a unique Zeno execution $\chi^{\mathrm{H}_{\mathbf{L}}}$ of $\mathrm{H}_{\mathbf{L}}$ with $c_{0}\left(\tau_{0}\right)=\left(q_{0}, \dot{q}_{0}\right)$.

Note that the theorem only gave sufficient conditions for existence of Zeno behavior, and did not discuss necessary conditions. In the next section, we show that the same conditions are sufficient for stability of Zeno equilibria, and also provide necessary conditions for stability.

\section{Examples}

We now introduce two examples of mechanical systems that will serve for demonstration of the results throughout the paper.

1) Example 1 (Ball): The first running example of this paper is a planar model of a ball bouncing on a sinusoidal surface [cf. Fig. 1(a)]. The ball is modeled as a point mass $m$. In this case, $\mathbf{B}=\left(Q_{\mathbf{B}}, L_{\mathbf{B}}, h_{\mathbf{B}}\right)$, where $Q_{\mathbf{B}}=\mathbb{R}^{2}$. The configuration is the position of the ball $q=(x, y)$, and the Lagrangian is given by $L_{\mathbf{B}}(x, \dot{x})=1 / 2 m\|\dot{q}\|^{2}-m g y$, where $g$ is the gravitational acceleration. Finally, the sinusoidal surface is represented by the constraint function $h_{\mathbf{B}}(q)=y-\sin (x) \geq 0$. So, for this example, there are trivial dynamics and a nontrivial constraint function.

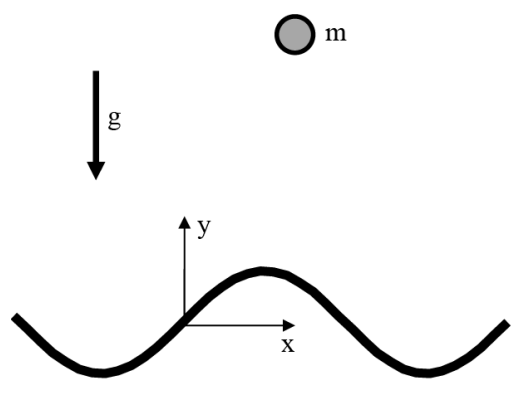

(a)

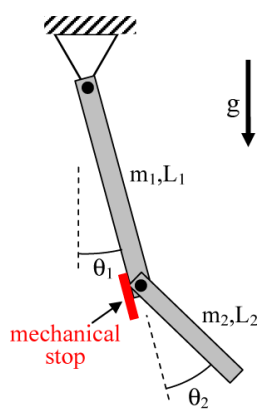

(b)
Fig. 1. (a) Bouncing ball on a sinusoidal surface (b) Double pendulum.

Note that from the hybrid Lagrangian $\mathbf{B}=\left(Q_{\mathbf{B}}, L_{\mathbf{B}}, h_{\mathbf{B}}\right)$, we obtain a hybrid system $\mathrm{H}_{\mathbf{B}}=\left(D_{\mathbf{B}}, G_{\mathbf{B}}, R_{\mathbf{B}}, f_{\mathbf{B}}\right)$. The set of Zeno equilibria for this hybrid system are given by

$$
Z=\left\{(x, y, \dot{x}, \dot{y}) \in G_{\mathbf{B}}: \dot{y}-\dot{x} \cos (x)=0\right\} .
$$

Physically, this set corresponds to states at which the ball touches the sinusoidal surface and slides along it.

2) Example 2 (Double Pendulum): Our second running example is a constrained double pendulum with a mechanical stop [cf. Fig. 1(b)]. The double pendulum consists of two rigid links of masses $m_{1}, m_{2}$, lengths $L_{1}, L_{2}$, and uniform mass distribution, which are attached by passive joints, while a mechanical stop dictates the range of motion of the second link. The example serves as a simplified model of a leg with a passive knee and a mechanical stop, which is widely investigated in the robotics literature in the context of passive dynamics of bipedal walkers with knees (cf. [28], [37]). In this case, $\mathbf{P}=$ $\left(Q_{\mathbf{P}}, L_{\mathbf{P}}, h_{\mathbf{P}}\right)$, where $Q_{\mathbf{P}}=\mathbb{S}^{1} \times \mathbb{S}^{1}, q=\left(\theta_{1}, \theta_{2}\right)$, and $\mathbb{S}^{1}$ denotes the unit circle, used for coordinates describing angles. The Lagrangian is given by $L_{\mathbf{P}}(q, \dot{q})=1 / 2 \dot{q}^{T} M(q) \dot{q}+$ $\left(1 / 2 m_{1} L_{1}+m_{2} L_{1}\right) g \cos \theta_{1}+1 / 2 m_{2} L_{2} g \cos \left(\theta_{1}+\theta_{2}\right)$, with the elements $M_{i j}$ of the $2 \times 2$ inertia matrix $M(q)$ given by $M_{11}=m_{1} L_{1}^{2} / 3+m_{2}\left(L_{1}^{2}+L_{2}^{2} / 3+L_{1} L_{2} \cos \theta_{2}\right), M_{12}=$ $M_{21}=m_{2}\left(3 L_{1} L_{2} \cos \theta_{2}+2 L_{2}^{2}\right) / 6, M_{22}=m_{2} L_{2}^{2} / 3$. Finally, the constraint that represents the mechanical stop is given by $h_{\mathbf{P}}(q)=\theta_{2} \geq 0$. So, for this example, there are nontrivial dynamics and a trivial constraint function.

Note that from the hybrid Lagrangian $\mathbf{P}=\left(Q_{\mathbf{P}}, L_{\mathbf{P}}, h_{\mathbf{P}}\right)$ we obtain a hybrid system $\mathrm{H}_{\mathbf{P}}=\left(D_{\mathbf{P}}, G_{\mathbf{P}}, R_{\mathbf{P}}, f_{\mathbf{P}}\right)$. The set of Zeno equilibria for this hybrid system are given by $Z=$ $\left\{\left(\theta_{1}, \theta_{2}, \dot{\theta}_{1}, \dot{\theta}_{2}\right) \in D_{\mathbf{P}}: \theta_{2}=0, \dot{\theta}_{2}=0\right\}$. That is, the set of Zeno equilibria are the set of points where the lower link is "locked."

\section{StabiLity OF Zeno EQuilibria}

In this section, we present and prove the first main result of this paper: conditions for the stability of Zeno equilibria. In particular, we introduce a type of stability that Zeno equilibria in SLHS can display: bounded-time local stability (BTLS). We show that the same conditions on the coefficient of restitution and the second derivative of the unilateral constraint function as in Theorem 1 imply this type of stability, and also give necessary conditions for stability. 
Definition 4: Let $x^{*}=\left(q^{*}, \dot{q}^{*}\right)$ be a Zeno equilibrium point of a simple Lagrangian hybrid system $\mathrm{H}_{\mathbf{L}}$. Then $x^{*}$ is defined as bounded-time locally stable (BTLS) if for each open neighborhood $U \subseteq T Q$ of $x^{*}$ and $\epsilon_{t}>0$, there exists another open neighborhood $W$ of $x^{*}$, such that for every initial conditions $c_{0}\left(\tau_{0}\right) \in W \cap D_{\mathbf{L}}$, the corresponding execution $\chi^{\mathrm{H}_{\mathbf{L}}}$ is Zeno, and satisfies $c_{i}(t) \in U$ for all $t \in I_{i}$ and $i \in \Lambda$, while its Zeno time satisfies $\tau_{\infty}-\tau_{0}<\epsilon_{t}$.

Remark: Note that this definition resembles the classical criterion of Lyapunov stability [26], with the two additions that only initial conditions that satisfy the constraint are allowed, and that finite-time convergence to the constraint surface is required. It is also similar in spirit to the criterion of uniform Zeno stability in [15], which is defined for hybrid systems whose dynamics is set-valued, and requires Zeno convergence of solutions to a compact set in state space. This stability notion is used in [15] for the special subclass of Lagrangian hybrid systems with isolated Zeno equilibrium points [21], whereas in our setup the Zeno equilibria are nonisolated and form a noncompact set.

\section{A. Statement of the Stability Conditions}

We now present the first result of the paper: conditions for bounded-time local stability of Zeno equilibria in simple Lagrangian hybrid systems.

Theorem 2: Let $x^{*}=\left(q^{*}, \dot{q}^{*}\right)$ be a Zeno equilibrium point of a simple Lagrangian hybrid system $\mathrm{H}_{\mathbf{L}}$, and assume that $e<1$. Then the following two conditions hold:

(i) If $\ddot{h}\left(q^{*}, \dot{q}^{*}\right)<0$, then $x^{*}$ is BTLS .

(ii) If $\ddot{h}\left(q^{*}, \dot{q}^{*}\right)>0$, then $x^{*}$ is not BTLS.

Remark: Note that the gap between the necessity and sufficiency conditions in this theorem can be intuitively viewed as being analogous to standard linearization results in continuous-time dynamical systems theory. In particular, in the case of linearizing a nonlinear system about an equilibrium point, if the real parts of the eigenvalues of the Jacobian are negative it implies stability, if one of the eigenvalues has positive real part it implies instability and if one of the real parts is zero it is not possible to determine stability or instability [40]. Therefore, in the case of Theorem $2, \ddot{h}\left(q^{*}, \dot{q}^{*}\right)$ plays the role of the "real part of an eigenvalue"; if it negative, there is stability, if it is positive there is instability, and if it is zero, an assessment of stability cannot be given in general.

1) Setup for the Proof of Theorem 2: For part (i) of Theorem 2 , we not only prove the existence of the neighborhood $W$ for given $U$, but also provide an explicit relation between $W$ and $U$. For the sake of concreteness and simplicity, we use a local coordinate chart for small neighborhoods of $x^{*}$. Therefore, we can identify both $q$ and $\dot{q}$ with elements of $\mathbb{R}^{n}$, and use the induced Euclidean norm $\|\cdot\|$ to define neighborhoods of $x^{*}=$ $\left(q^{*}, \dot{q}^{*}\right)$ as $N\left(\epsilon_{q}, \epsilon_{v}\right)=\left\{(q, \dot{q}) \in D_{\mathbf{L}}:\left\|q-q^{*}\right\|<\epsilon_{q}, \| \dot{q}-\right.$ $\left.\dot{q}^{*} \|<\epsilon_{v}\right\}$. Using this notation, for a given $U$ there exist $\epsilon_{q}$ and $\epsilon_{v}$ such that $N\left(\epsilon_{q}, \epsilon_{v}\right) \subseteq U$. Assuming that $e<1$ and $\ddot{h}\left(q^{*}, \dot{q}^{*}\right)<0$, our goal is to construct a neighborhood $W=$ $N\left(\delta_{q}, \delta_{v}\right)$ that satisfies the requirements given in Definition 4.

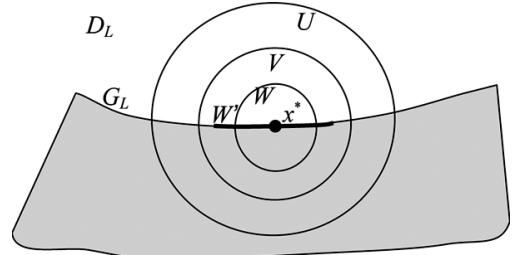

Fig. 2. Illustration of the neighborhoods $U, V, W$ and $W^{\prime}$ of $x^{*}$.

\section{Proof of the Stability Conditions}

The rest of this section proves Theorem 2 by stages through a series of lemmas. Before presenting these lemmas, we will first give a general outline of the proof. In particular, the proof of part (i) of Theorem 2 is divided into three steps.

1) We define an intermediate neighborhood $V \subset U$, such that solutions of the hybrid system that stay within $V$ are converging to the set $Z$ of Zeno equilibria.

2) We define another neighborhood $W^{\prime} \subset G_{\mathbf{L}} \cap V$ which lies on the guard $G_{\mathbf{L}}$, such that for any execution, if at the first impact time $c_{0}\left(\tau_{1}\right)$ lies in $W^{\prime}$ then it is a Zeno execution whose solution is guaranteed to stay within $V$.

3) We construct the neighborhood $W$, such that any execution with initial conditions $c_{0}\left(\tau_{0}\right) \in W$ is guaranteed to satisfy $c_{0}\left(\tau_{1}\right) \in W^{\prime}$, and thus it is a Zeno execution, and the solution stays within $\mathrm{U}$, as required. An illustration of these neighborhoods appear in Fig. 2.

We now formally proceed through these steps in order to complete the proof of Theorem 2 .

2) Step 1: We begin by defining the intermediate neighborhood $V=N\left(\epsilon_{q}^{\prime}, \epsilon_{v}^{\prime}\right)$, where $\epsilon_{q}^{\prime}<\epsilon_{q}$ and $\epsilon_{v}^{\prime}<\epsilon_{v}$ are chosen so that for $a_{\min }=-\max _{(q, \dot{q}) \in V} \ddot{h}(q, \dot{q})$ and $a_{\max }=$ $-\min _{(q, \dot{q}) \in V} \ddot{h}(q, \dot{q})$, the following conditions hold:

$$
a_{\max }>a_{\min }>0 \text { and } e \gamma<1 \text {, where } \gamma=\sqrt{\frac{a_{\max }}{a_{\min }}} .
$$

Note that the fact that $e<1$ and $\ddot{h}\left(q^{*}, \dot{q}^{*}\right)<0$, along with the continuity of $\ddot{h}(q, \dot{q})$, imply that such $\epsilon_{q}^{\prime}, \epsilon_{v}^{\prime}$ exist. This definition of $V$ implies that when $(q(t), \dot{q}(t)) \in V$, the timeevolution of the constraint function $h(q(t))$ satisfies the secondorder differential inclusion

$$
\ddot{h}(q(t), \dot{q}(t)) \in\left[-a_{\max },-a_{\min }\right] .
$$

For simplicity of notation, for an execution $\chi^{\mathrm{H}_{\mathrm{L}}}$ let us denote $v_{i}^{-}=d h\left(q_{i-1}\left(\tau_{i}\right)\right) \dot{q}_{i-1}\left(\tau_{i}\right)$ and $v_{i}^{+}=d h\left(q_{i}\left(\tau_{i}\right)\right) \dot{q}_{i}\left(\tau_{i}\right)$, which are the discrete sequences of pre- and post-impact velocities $\dot{h}_{\text {at }}$ the collision times $\tau_{i}$. Note that the impact law (4) implies that $v_{i}^{+}=-e v_{i}^{-}$. Also, let $\Delta \tau_{i}=\tau_{i}-\tau_{i-1}$ denote the time difference between two consecutive impacts. The following lemma states that in a part of an execution where the solution stays within $V$, the series of $v_{i}^{-}$and $\Delta \tau_{i}$ are decaying.

Lemma 1: Let $x^{*}=\left(q^{*}, \dot{q}^{*}\right)$ be a Zeno equilibrium point of a simple Lagrangian hybrid system such that $\ddot{h}\left(q^{*}, \dot{q}^{*}\right)<0$ and $e<1$, and let $V=N\left(\epsilon_{q}^{\prime}, \epsilon_{v}^{\prime}\right)$ be a neighborhood of $x^{*}$ that satisfies the conditions in (6). Then for any execution $\chi^{\mathrm{H}_{\mathrm{L}}}$ 
such that $c_{i}(t) \in V$ for all $t \in I_{i}$ and $i \in\{1 \ldots k\} \subseteq \Lambda$, the discrete-time series of $v_{i}^{-}$and of $\Delta \tau_{i}$ satisfy

$$
\begin{aligned}
\frac{e}{\gamma}\left|v_{i}^{-}\right| & \leq\left|v_{i+1}^{-}\right| \leq e \gamma\left|v_{i}^{-}\right| \quad \text { for } i=1 \ldots k \\
\Delta \tau_{i} & \leq \frac{2 e\left|v_{i}^{-}\right|}{a_{\min }} \quad \text { for } i=1 \ldots k
\end{aligned}
$$

The proof of Lemma 1, which utilizes methods from optimal control to derive bounds on the solution, appears in the Appendix. The key idea of the proof is based on the fact that $h(q(t))$ along the solution $c_{i}(t)$ must satisfy the differential inclusion (7). Therefore, finding bounds on all possible solutions of (7) will also imply a bound on the unknown solution $c_{i}(t)$. The lemma implies that the sequence of pre-impact velocities is bounded by a geometric series as $\left|v_{i}^{-}\right| \leq\left|v_{1}^{-}\right|(e \gamma)^{i-1}$ for $i=$ $1 \ldots k$. The condition $e \gamma<1$ in (6) then implies that the series of $v_{i}^{-}$is bounded by a decaying geometric series. Moreover, in the special case where $c_{i}(t) \in V$ at all times, (8) and (9) imply that there is an infinite number of impacts occurring in finite time, that is, $\chi^{\mathrm{H}_{\mathbf{L}}}$ is a Zeno execution.

Note that one can obtain alternative conditions on $a_{\min }, a_{\max }$ and $e$ that guarantee the decaying of $v_{i}^{-}$by using elementary calculus and direct integration of (7). However, these conditions are much more conservative than those given in (6). In order to derive the tight bound (8) for $v_{i+1}^{-}$over all feasible solutions of (7), one has to utilize methods of optimal control (or, equivalently, calculus of variations), as detailed in the Appendix.

3) Step 2: As the next step towards computing the neighborhood $\mathrm{W}$, we define the relative neighborhood $W^{\prime} \subset G_{\mathbf{L}} \cap V$, of initial conditions on the guard $G_{\mathbf{L}}$ (i.e., corresponding to an impact), such that for any execution with initial conditions in $W^{\prime}$, the solution stays within $V$.

In order to construct $W^{\prime}$ for given neighborhoods $U$ and $V$, we first define the scalars

$$
\begin{aligned}
\beta & =\left\|\dot{q}^{*}\right\|+\epsilon_{v}^{\prime} \\
\eta & =\max _{(q, \dot{q}) \in V} \frac{\left\|M^{-1}(q) d h(q)^{T}\right\|}{d h(q) M(q) d h(q)^{T}} \\
\zeta & =\max _{(q, \dot{q}) \in V}\left\|M^{-1}(q)(C(q, \dot{q}) \dot{q}+N(q))\right\| .
\end{aligned}
$$

The following lemma completes the definition of $W^{\prime}$.

Lemma 2: Let $x^{*}=\left(q^{*}, \dot{q}^{*}\right)$ be a Zeno equilibrium point of a simple Lagrangian hybrid system $\mathrm{H}_{\mathbf{L}}$ such that $\ddot{h}\left(q^{*}, \dot{q}^{*}\right)<0$ and $e<1$, and let $V=N\left(\epsilon_{q}^{\prime}, \epsilon_{v}^{\prime}\right)$ be a neighborhood of $x^{*}$ that satisfies (6). For a given $\epsilon_{t}^{\prime}>0$, let $W^{\prime}$ be the relative neighborhood defined as follows:

$$
\begin{aligned}
W^{\prime}=\left\{(q, \dot{q}) \in T Q: h(q)=0,\left\|q-q^{*}\right\| \leq \delta_{q}^{\prime},\right. \\
\left.\left\|\dot{q}-\dot{q}^{*}\right\| \leq \delta_{v}^{\prime} \text { and }-v_{1 \max }<d h(q) \dot{q} \leq 0\right\}
\end{aligned}
$$

such that $\delta_{q}^{\prime}, \delta_{v}^{\prime}$ and $v_{1 \max }$ satisfy the conditions

$$
\begin{aligned}
& \delta_{q}^{\prime}<\epsilon_{q}^{\prime}, \delta_{v}^{\prime}<\epsilon_{v}^{\prime} \text { and } v_{1 \max }<\min \left\{\kappa_{1}, \kappa_{2}, \kappa_{3}\right\} \\
& \kappa_{1}=\frac{a_{\min }(1-e \gamma)}{2 e} \epsilon_{t}^{\prime} \\
& \kappa_{2}=\frac{a_{\min }(1-e \gamma)}{2 e \beta}\left(\epsilon_{q}^{\prime}-\delta_{q}^{\prime}\right)
\end{aligned}
$$

$$
\kappa_{3}=\frac{\left(\epsilon_{v}^{\prime}-\delta_{v}^{\prime}\right)}{\left(\frac{(1+e) \eta}{1-e \gamma}+\frac{2 e \zeta}{a_{\min }(1-e \gamma)}\right)} .
$$

Then each execution $\chi^{\mathrm{H}_{\mathrm{L}}}$ such that $c_{0}\left(\tau_{1}\right) \in W^{\prime}$ is Zeno and satisfies $c_{i}(t) \in V$ for all $t \in I_{i}$ and $i \geq 1$. Moreover, the corresponding Zeno time satisfies $\tau_{\infty}-\tau_{1}<\epsilon_{t}^{\prime}$.

Proof: Let us denote $c_{0}\left(\tau_{1}\right)=\left(q_{1}, \dot{q}_{1}\right)$ and treat this as the initial condition of the execution, hence the first time interval is $I_{0}=\left\{\tau_{1}\right\}$. Note that, by construction of $W^{\prime}$, the initial condition satisfies $c_{0}\left(\tau_{1}\right) \in V$. We prove the lemma by assuming that the solution does not stay within $V$ at all times, and showing that this leads to a contradiction. Let $t^{\prime}$ be the minimal time such that $c_{i^{\prime}}\left(t^{\prime}\right) \notin V$, where $t^{\prime} \in I_{i^{\prime}}$, and $i^{\prime} \in \Lambda$. This implies that $c_{i}(t) \in V$ for all $i=1 \ldots i^{\prime}-1$, and that $c_{i^{\prime}}(t) \in V$ for all $t \in I_{i^{\prime}}$ such that $t<t^{\prime}$. We now invoke the results of Lemma 1 to derive bounds on $c_{i^{\prime}}\left(t^{\prime}\right)$, and show that it must lie within $V$. First, we derive an upper bound for the time $t^{\prime}$. Using Lemma 1 and substituting $k=i^{\prime}-1$, the time differences $\Delta \tau_{i}$ must satisfy (9) for $i=1 \ldots i^{\prime}-1$. This implies that

$$
\tau_{i^{\prime}}=\tau_{1}+\sum_{i=1}^{i^{\prime}-1} \Delta \tau_{i} \leq \tau_{1}+\frac{2 e\left|v_{1}^{-}\right|}{a_{\min }} \sum_{i=1}^{i^{\prime}-1}(e \gamma)^{i-1} .
$$

Since $c_{i}(t) \in V$ for $t \in\left[\tau_{i}, t^{\prime}\right)$, the acceleration $\ddot{h}$ satisfies the differential inclusion (7). Thus, direct integration gives the bound $h\left(q_{i^{\prime}}(t)\right)<v_{i^{\prime}}^{+}\left(t-\tau_{i^{\prime}}\right)-a_{\min }\left(t-\tau_{i^{\prime}}\right)^{2} / 2$, and the inequality $h(q) \geq 0$ then results in $\left(t^{\prime}-\tau_{i^{\prime}}\right) \leq 2 v_{i^{\prime}}^{+} / a_{\min }$. Using (9) and the fact that $v_{i^{\prime}}^{+}=e v_{i^{\prime}}^{-}$, one obtains an upper bound on $t^{\prime}$ as

$$
t^{\prime} \leq \tau_{1}+\frac{2 e\left|v_{1}^{-}\right|}{a_{\min }} \sum_{i=1}^{i^{\prime}}(e \gamma)^{i}<\frac{2 e\left|v_{1}^{-}\right|}{a_{\min }(1-e \gamma)} .
$$

We now derive a bound on $q_{i^{\prime}}\left(t^{\prime}\right)$. By definition of the impact law (4), the $q$-component of the solution $c_{i}(t)$ does not change at the impact times, i.e., $q_{i}\left(\tau_{i+1}\right)=q_{i+1}\left(\tau_{i+1}\right)$ for all $i \in \Lambda$. Moreover, the definition of $\beta$ in (10) implies that if $c_{i}(t) \in V$ then $\left\|\dot{q}_{i}(t)\right\|<\beta$. Since this holds for all $i<i^{\prime}$ and for $i=i^{\prime}$ and $t<t^{\prime}$, the change in $q$ is bounded by $\left\|q_{i^{\prime}}\left(t^{\prime}\right)-q_{1}\right\|<\beta\left(t^{\prime}-\tau_{1}\right)$. Note that, by construction of $W^{\prime}$, $q_{1}$ satisfies $\left\|q_{1}-q^{*}\right\| \leq \delta_{q}^{\prime}$. Using this fact and the bound on $t^{\prime}$ in (13), the triangle inequality implies that $\left\|q_{i^{\prime}}\left(t^{\prime}\right)-q^{*}\right\|<$ $\delta_{q}^{\prime}+\beta 2 e\left|v_{1}^{-}\right| / a_{\min }(1-e \gamma)$. By construction of $W^{\prime}$ in (12), the requirement $v_{1 \max }<\kappa_{2}$ then implies that $\left\|q_{i^{\prime}}\left(t^{\prime}\right)-q^{*}\right\|<\epsilon_{q}^{\prime}$.

Next, we derive a bound on $\dot{q}_{i^{\prime}}\left(t^{\prime}\right)$. The change in $\dot{q}$ during the execution can be decomposed into its discrete and continuous parts, as follows. Let us denote $\Delta_{i}^{d}=\dot{q}_{i}\left(\tau_{i}\right)-\dot{q}_{i-1}\left(\tau_{i}\right)$ and $\Delta_{i}^{c}=\dot{q}_{i}\left(\tau_{i+1}\right)-\dot{q}_{i}\left(\tau_{i}\right)$. Therefore, one can express $\dot{q}_{i^{\prime}}\left(\tau_{i^{\prime}}\right)$ as

$$
\dot{q}_{i^{\prime}}\left(\tau_{i^{\prime}}\right)=\dot{q}_{1}+\Delta_{1}^{d}+\Delta_{1}^{c} \cdots+\Delta_{i^{\prime}-1}^{d}+\Delta_{i^{\prime}-1}^{c}+\Delta_{i}^{d} .
$$

We now use the fact that $c_{i}(t) \in V$ for all $t \in I_{i}$ and $i=$ $1 \ldots i^{\prime}$ to derive bounds on $\Delta_{i}^{d}$ and $\Delta_{i}^{c}$. The discrete change in $\dot{q}$ due to a single impact at time $\tau_{i}$ is given in (4). When $c_{i}\left(\tau_{i}\right) \in V$, it is bounded by $\left\|\Delta_{i}^{d}\right\| \leq(1+e) \eta\left|v_{i}^{-}\right|$, where $\eta$ is defined in (10). The change in $\dot{q}$ during the time interval $I_{i}$ is bounded by $\left\|\Delta_{i}^{d}\right\| \leq \zeta \Delta \tau_{i}$, where $\zeta$, defined in (10), is the maximum norm of $\ddot{q}$ in $V$. Similarly, the change in $\dot{q}$ during the time interval $\left[\tau_{i^{\prime}}, t^{\prime}\right)$ satisfies $\left\|\dot{q}_{i^{\prime}}\left(t^{\prime}\right)-\dot{q}_{i^{\prime}}\left(\tau_{i^{\prime}}\right)\right\| \leq \zeta\left(t^{\prime}-\tau_{i^{\prime}}\right)$. 
Using these bounds together with the bound on $t^{\prime}$ in (13), the bounds (8), (9) from Lemma 1, and the decomposition (14), the triangle inequality implies a bound on $\dot{q}_{i^{\prime}}\left(t^{\prime}\right)$ as

$$
\begin{aligned}
\left\|\dot{q}_{i^{\prime}}\left(t^{\prime}\right)-q_{1}\right\| \leq & \sum_{i=1}^{i^{\prime}}(1+e) \eta\left|v_{i}^{-}\right| \\
& +\sum_{i=1}^{i^{\prime}-1} \zeta \Delta \tau_{i}+\zeta\left(t^{\prime}-\tau_{i^{\prime}}\right) \\
\leq & (1+e) \eta\left|v_{1}^{-}\right| \sum_{i=1}^{i^{\prime}}(e \gamma)^{i-1} \\
& +\zeta\left(t^{\prime}-\tau_{1}\right) \\
< & \left(\frac{(1+e) \eta}{1-e \gamma}+\frac{2 e \zeta}{a_{\min }(1-e \gamma)}\right)\left|v_{1}^{-}\right| .
\end{aligned}
$$

Note that, by construction of $W^{\prime}, \dot{q}_{1}$ satisfies $\left\|\dot{q}_{1}-\dot{q}^{*}\right\| \leq \delta_{v}^{\prime}$. Using the triangle inequality and the bound (15), the requirement $v_{1 \max }<\kappa_{3}$ in (12) then implies that $\left\|\dot{q}_{i^{\prime}}\left(t^{\prime}\right)-\dot{q}^{*}\right\|<\epsilon_{v}^{\prime}$. Combining this with the bound obtained for $q_{i^{\prime}}\left(t^{\prime}\right)$, we conclude that $c_{i^{\prime}}\left(t^{\prime}\right) \in V$, in contradiction to the original assumption.

Finally, since $c_{i^{\prime}}\left(t^{\prime}\right) \in V$ for all $t \in I_{i}$ and $i \in \Lambda$, the lower bound in (8) in Lemma 1 implies that $v_{i}^{-}$is always strictly nonzero for any $i \in \Lambda$. Therefore, the solution $c_{i^{\prime}}\left(t^{\prime}\right)$ does not reach the constraint surface $Z$ at any time $\tau_{i}$ after a finite number of impacts. Instead, it converges asymptotically to $Z$ after an infinite number of impacts, hence $\chi^{\mathrm{H}_{\mathrm{L}}}$ must be a Zeno execution. Moreover, using the bound (9) in Lemma 1, the Zeno time is bounded by

$$
\begin{aligned}
\tau_{\infty} & =\tau_{1}+\sum_{i=1}^{\infty} \Delta \tau_{i} \leq \tau_{1}+\sum_{i=1}^{\infty} \frac{2 e\left|v_{1}^{-}\right|}{a_{\min }}(e \gamma)^{i=1} \\
& =\tau_{1}+\frac{2 e\left|v_{1}^{-}\right|}{a_{\min }(1-e \gamma)} .
\end{aligned}
$$

The requirement that $v_{1 \max }<\kappa_{1}$ in (12) then verifies that $\tau_{\infty}-$ $\tau_{1}<\epsilon_{t}^{\prime}$, as desired.

4) Step 3: At this final stage, for a given $\epsilon_{t}^{\prime \prime}>0$, we define the neighborhood $W$ as $W=N\left(\delta_{q}, \delta_{v}\right)$, where $\delta_{q}<\delta_{q}^{\prime}$ and $\delta_{q}<\delta_{q}^{\prime}$ satisfy

(i) $\frac{d h(q) \dot{q}+\sqrt{(d h(q) \dot{q})^{2}-a_{\min } h(q)}}{a_{\min }}<\min \left\{\frac{\delta_{q}^{\prime}-\delta_{q}}{\beta}, \frac{\delta_{v}^{\prime}-\delta_{v}}{\zeta}, \epsilon_{t}^{\prime \prime}\right\}$

$$
\left(2 h(q)+\frac{(d h(q) \dot{q})^{2}}{a_{\min }}\right) a_{\max }<\left(v_{1 \max }\right)^{2}
$$

for all $(q, \dot{q}) \in N\left(\delta_{q}, \delta_{v}\right) \cap D_{\mathbf{L}}$, where $\beta$ and $\zeta$ are defined in (10).

Note that since $h\left(q^{*}\right)=0$ and $d h\left(q^{*}\right) \dot{q}^{*}=0$, continuity of $h(q)$ and $d h(q)$ imply that such $\delta_{q}, \delta_{v}$ exist. The following lemma states that if the initial condition are within $W$, then at the first impact time, $c_{0}\left(\tau_{1}\right)$ lies within $W^{\prime}$.

Lemma 3: Let $x^{*}=\left(q^{*}, \dot{q}^{*}\right)$ be a Zeno equilibrium point of a simple Lagrangian hybrid system $\mathrm{H}_{\mathbf{L}}$ such that $\ddot{h}\left(q^{*}, \dot{q}^{*}\right)<0$ and $e<1$, and let $V, W^{\prime}$ and $W$ be the neighborhoods of $x^{*}$ defined in (6), (11) and (16) respectively. Then each execution $\chi^{\mathrm{H}_{\mathbf{L}}}$ such that $c_{0}\left(\tau_{0}\right) \in W \cap D_{\mathbf{L}}$ satisfies $c_{0}(t) \in V$ for $t \in I_{0}$, and $c_{0}\left(\tau_{1}\right) \in W^{\prime}$ and $\tau_{1}-\tau_{0}<\epsilon_{t}^{\prime \prime}$.

Proof: From the definition of $W$ and $V$, it is clear that the initial condition satisfies $c_{0}\left(\tau_{0}\right) \in V$. First, in order to prove that $c_{0}(t) \in V$ for all $t \in I_{0}$, we assume that there exists a time $t^{\prime} \in I_{0}$ such that $c_{0}\left(t^{\prime}\right) \notin V$, and show that this leads to a contradiction, in a manner similar to the proof of Lemma 2. Since $c_{0}\left(\tau_{0}\right) \in V$, we take $t^{\prime}$ as the minimal time such that $c_{0}\left(t^{\prime}\right) \notin V$, so that $c_{0}(t) \in V$ for all $t \in\left[\tau_{0}, t^{\prime}\right)$. This implies bounds on $q_{0}\left(t^{\prime}\right)$ and $\dot{q}_{0}\left(t^{\prime}\right)$ as

$$
\begin{aligned}
& \left\|q_{0}\left(t^{\prime}\right)-q_{0}\left(\tau_{0}\right)\right\| \leq \beta\left(t^{\prime}-\tau_{0}\right) \\
& \left\|\dot{q}_{0}\left(t^{\prime}\right)-\dot{q}_{0}\left(\tau_{0}\right)\right\| \leq \zeta\left(t^{\prime}-\tau_{0}\right) .
\end{aligned}
$$

Moreover, $h(q(t))$ satisfies the differential inclusion (7) for $t \in$ $\left[\tau_{0}, t^{\prime}\right)$. Thus, integrating (7) twice gives an upper bound for $h\left(q\left(t^{\prime}\right)\right)$ as $h\left(q\left(t^{\prime}\right)\right) \leq h_{0}+v_{0}\left(t-\tau_{0}\right)-a_{\min }\left(t-\tau_{0}\right)^{2} / 2$, where $h_{0}=h\left(q_{0}\left(\tau_{0}\right)\right)$ and $v_{0}=d h\left(q_{0}\left(\tau_{0}\right)\right) \dot{q}_{0}\left(\tau_{0}\right)$. Since $h\left(q\left(t^{\prime}\right)\right) \geq$ 0 , one obtains a bound on $t^{\prime}$ as

$$
t^{\prime}-\tau_{0} \leq \frac{v_{0}+\sqrt{v_{0}^{2}+2 a_{\min } h_{0}}}{a_{\min }} .
$$

Substituting (18) into (17), condition (i) in (16) gives $\| q_{0}\left(t^{\prime}\right)-$ $q_{0}\left(\tau_{0}\right) \| \leq \delta_{q}^{\prime}-\delta_{q}$ and $\left\|\dot{q}_{0}\left(t^{\prime}\right)-\dot{q}_{0}\left(\tau_{0}\right)\right\| \leq \delta_{v}^{\prime}-\delta_{v}$. Using the fact that $\left(q_{0}\left(\tau_{0}\right), \dot{q}_{0}\left(\tau_{0}\right)\right) \in W=N\left(\delta_{q}, \delta_{v}\right)$, the triangle inequality implies that $\left(q_{0}\left(t^{\prime}\right), \dot{q}_{0}\left(t^{\prime}\right)\right) \in N\left(\delta_{q}^{\prime}, \delta_{v}^{\prime \prime}\right) \subset V$, in contradiction to the original assumption. Next, since $c_{0}(t) \in V$ for all $t \in I_{0}$, the differential inclusion (7) is satisfied for all $t \in I_{0}$. Similar to the derivation of (18), it can be shown that $\tau_{1}-\tau_{0}$ satisfies the same bound given in the right hand side of (18). Therefore, condition (i) in (16) also implies that $\tau_{1}-\tau_{0}<\epsilon_{t}^{\prime \prime}$. Using the same arguments as above with $\tau_{1}$ instead of $t^{\prime}$, it can be shown that $c_{0}\left(\tau_{1}\right) \in N\left(\delta_{q}^{\prime}, \delta_{v}^{\prime}\right)$. Moreover, using optimal control, it is shown in the Appendix that $v_{1}^{-}$satisfies

$$
\left|v_{1}^{-}\right| \leq \sqrt{\left(2 h_{0}+\frac{\left(v_{0}\right)^{2}}{a_{\min }}\right) a_{\max }} .
$$

Condition (ii) in (16) then implies that $\left|v_{1}^{-}\right|<v_{1 \max }$, hence $c_{0}\left(\tau_{1}\right)$ lies within $W^{\prime}$.

We now combine the results above to complete the proof of Theorem 2.

Proof of Theorem 2: First, we prove part (i). Assume that $e<1$ and $\ddot{h}\left(q^{*}, \dot{q}^{*}\right)<0$. For the given neighborhood $U$ and $\epsilon_{t}>0$, pick $\epsilon_{q}, \epsilon_{v}$ such that $N\left(\epsilon_{q}, \epsilon_{v}\right) \subseteq U$. Then choose $\epsilon_{q}^{\prime}<\epsilon_{q}$ and $\epsilon_{v}^{\prime}<\epsilon_{v}$ such that the neighborhood $V=N\left(\epsilon_{q}^{\prime}, \epsilon_{v}^{\prime}\right)$ satisfies (6). Next, choose $\epsilon_{t}^{\prime}<\epsilon_{t}$ and $\delta_{q}^{\prime}, \delta_{v}^{\prime}$ and $v_{1 \max }$ such that (12) is satisfied. The neighborhood $W^{\prime}$ is then defined in (11). Finally, choose $\epsilon_{t}^{\prime \prime}<\epsilon_{t}-\epsilon_{t}^{\prime}$, and $\delta_{q}<\delta_{q}^{\prime}, \delta_{v}<\delta_{v}^{\prime}$ that satisfy (16), and define $W=N\left(\delta_{q}, \delta_{v}\right)$. Consider an execution $\chi^{\mathrm{H}_{\mathbf{L}}}$ with initial conditions $c_{0}\left(\tau_{0}\right) \in W \cap D_{\mathbf{L}}$. Lemma 3 implies that $c_{0}(t) \in V$ for $t \in I_{0}$, and $c_{0}\left(\tau_{1}\right) \in W^{\prime}$ and $\tau_{1}-\tau_{0}<\epsilon_{t}^{\prime \prime}$. Lemma 2 then implies that $c_{i}(t) \in V$ for all $t \in I_{i}$ and $i \geq 1$, that $\chi^{\mathrm{H}_{\mathrm{L}}}$ is Zeno, and that $\tau_{\infty}-\tau_{1}<\epsilon_{t}^{\prime}$. Therefore, $\chi^{\mathrm{H}_{\mathrm{L}}}$ is Zeno, the Zeno time satisfies $\tau_{\infty}-\tau_{0}<\epsilon_{t}$, and the solution stays bounded within $V \subset U$ as desired.

We now prove part (ii) of the theorem in the case where $\ddot{h}\left(q^{*}, \dot{q}^{*}\right)>0$. First, choose $a_{0}>0$ such that $a_{0}<\ddot{h}\left(q^{*}, \dot{q}^{*}\right)$. Next, choose an open neighborhood $\bar{U}$ of $x^{*} \operatorname{such}$ that $\ddot{h}(q, \dot{q})>$ $a_{0}$ for any $(q, \dot{q}) \in \bar{U}$, and define $h_{\max }=\max \{h(q) \mid(q, \dot{q}) \in$ $\bar{U}\}$. Choose any initial condition $\left(q_{0}, \dot{q}_{0}\right) \in \bar{U} \cap D_{\mathbf{L}}$ such that $d h\left(q_{0}\right) \dot{q}_{0}>0$, and assume that the corresponding execution 
$\chi^{\mathrm{H}_{\mathbf{L}}}$ satisfies $c_{0}(t) \in \bar{U}$ for all $t \in I_{0}$. Then by construction, $h\left(q_{0}(t)\right)$ satisfies $\ddot{h}\left(q_{0}(t), \dot{q}_{0}(t)\right)>a_{0}$ for all $t \in I_{0}$, and its initial conditions are $h\left(q_{0}\left(\tau_{0}\right)\right) \geq 0$ and $\dot{h}\left(q_{0}\left(\tau_{0}\right), \dot{q}_{0}\left(\tau_{0}\right)\right)>0$. It can be easily shown that there exists a time $t^{\prime} \leq \tau_{0}+\sqrt{2 a_{0} h_{\max }}$ such that $h\left(q_{0}\left(t^{\prime}\right)\right)>h_{\max }$, and thus $\left(q_{0} \overline{\left(t^{\prime}\right.}\right), \dot{q}_{0}\left(t^{\prime}\right) \notin \bar{U}$. Therefore, the solution cannot be bounded within $\bar{U}$ by setting the initial conditions arbitrarily close to $x^{*}$, in contradiction with the definition of BTLS.

\section{COMPLETED LAGRANGIAN HybRID SySTEM}

In this section we present a method for carrying executions of Lagrangian hybrid systems beyond Zeno time, i.e., we formally define a "completed" Lagrangian hybrid system.

1) Overview of Completion Process: The motivation for completing hybrid system models is based upon the fact that despite the name "Zeno equilibrium," a Zeno point $x_{\infty}$ is not a physical equilibrium point, since it satisfies $f_{\mathbf{L}}\left(x_{\infty}\right) \neq 0$, and involves nonzero velocity. It was postulated in [5] that after the Zeno time, the system should switch to a holonomically constrained dynamical system. Note that this postulation is essentially a modeling paradigm, and as such, its correctness cannot be mathematically proven. However, we argue that this concept correctly captures the physical behavior in this class of mechanical systems.

The main observation of [5] is that at a Zeno point, the execution of the Lagrangian hybrid system converges to a limit point $\left(q_{\infty}, \dot{q}_{\infty}\right)$ that satisfies $h\left(q_{\infty}\right)=0$ and $d h\left(q_{\infty}\right) \dot{q}_{\infty}=0$. This limit point lies on the constraint surface $h^{-1}(0)=\{q \in Q$ : $h(q)=0\}$, and its velocity vector is tangent to this surface. Since in Lagrangian hybrid systems the unilateral constraint $h(q) \geq 0$ usually represents a mechanical contact, it is hypothesized in [5] that once such a contact is re-established via a Zeno execution, it is then maintained by a constraining force. This behavior is captured by the formulation of a holonomically constrained dynamical system whose trajectories are constrained to the surface $h^{-1}(0)$, where the constraint is maintained by a Lagrange multiplier $\lambda(t)$ representing the physical contact force. An important fact that was overlooked in [5] is that, in such systems, a contact force is often also constrained to be nonnegative, thus eliminating tension or adhesion forces. Under this assumption, our completed model suggests that at a zero-crossing event of the constraint force, the constrained system switches back to the hybrid system. Physically, this event corresponds to a contact breakage and separation.

2) Constrained Dynamical Systems: We now define the holonomically constrained dynamical system $\mathrm{D}_{\mathbf{L}}$ associated with the hybrid Lagrangian L. For such systems, the constrained equations of motion can be obtained from the equations of motion for the unconstrained system (2), and are given by (cf. [30])

$$
M(q) \ddot{q}+C(q, \dot{q}) \dot{q}+N(q)=d h(q)^{T} \lambda
$$

where $\lambda$ is the Lagrange multiplier which represents the contact force. Differentiating the constraint $h(q(t))=0$ twice with respect to time and substituting the solution for $\ddot{q}$ in (20), the solution for the constraint force is obtained as follows:

$\lambda(q, \dot{q})=\left(d h(q) M(q)^{-1} d h(q)^{T}\right)^{-1}$

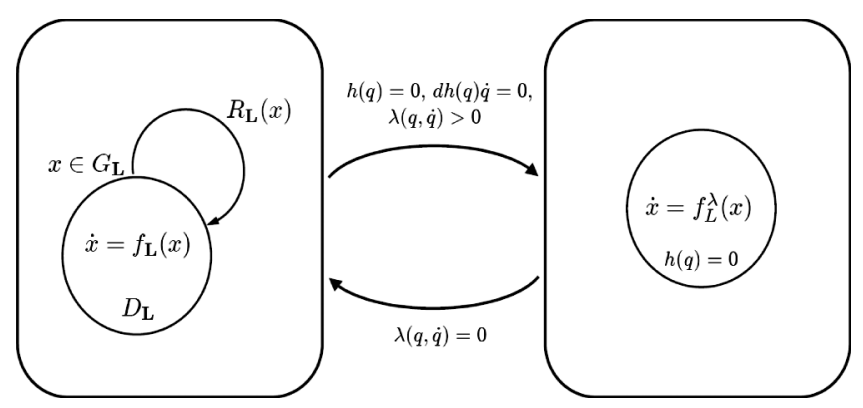

Fig. 3. Graphical representation of a completed hybrid system.

$$
\left(d h(q) M(q)^{-1}(C(q, \dot{q}) \dot{q}+N(q))-\dot{q}^{T} H(q) \dot{q}\right) .
$$

From the constrained equations of motion, for $x=(q, \dot{q})$, we get the vector field

$\dot{x}=f_{L}^{\lambda}(x)=\left(\begin{array}{c}\dot{q} \\ -M(q)^{-1}\left(C(q, \dot{q}) \dot{q}+N(q)-d h(q)^{T} \lambda(q, \dot{q})\right)\end{array}\right)$.

Note that $f_{L}^{\lambda}$ defines a vector field on the manifold $\left.T Q\right|_{h^{-1}(0)}$, from which we obtain the dynamical system $\mathrm{D}_{\mathbf{L}}=\left(\left.T Q\right|_{h^{-1}(0)}, f_{L}^{\lambda}\right)$. For this dynamical system, $q(t)$ slides along the surface $h^{-1}(0)$ as long as the constraint force $\lambda$ is positive.

A constrained execution $\tilde{\chi}$ of $\mathrm{D}_{\mathbf{L}}$ is a pair $(\tilde{I}, \tilde{c})$ where $\tilde{I}=$ $\left[\tilde{\tau}_{0}, \tilde{\tau}_{f}\right] \subset \mathbb{R}$ if $\tilde{\tau}_{f}$ is finite and $\tilde{I}=\left[\tilde{\tau}_{0}, \tilde{\tau}_{f}\right) \subset \mathbb{R}$ if $\tilde{\tau}_{f}=\infty$ and $\tilde{c}: \tilde{I} \rightarrow T Q$, with $\tilde{c}(t)=(q(t), \dot{q}(t))$ a solution to the dynamical system $D_{\mathbf{L}}$ satisfying the following properties:

$$
\begin{aligned}
& \text { (i) } h\left(q\left(\tilde{\tau}_{0}\right)\right)=0 \\
& \text { (ii) } d h\left(q\left(\tilde{\tau}_{0}\right)\right) \dot{q}\left(\tilde{\tau}_{0}\right)=0 \\
& \text { (iii) } \lambda\left(q\left(\tilde{\tau}_{0}\right), \dot{q}\left(\tilde{\tau}_{0}\right)\right)>0 \\
& \text { (iv) } \tilde{\tau}_{f}=\min \{t \in \tilde{I}: \lambda(q(t), \dot{q}(t))=0\} .
\end{aligned}
$$

3) The Completed Hybrid Systems and Completed Executions: Using the notation and concepts introduced thus far, we now introduce the notion of a completed hybrid system, denoted $\overline{\mathrm{H}}_{\mathbf{L}}$. Loosely speaking, the completed hybrid system has components of hybrid dynamics and constrained dynamics, and two-way transitions between them. Fig. 3 depicts a graphical representation of the completed system. In order to more formally define the solutions of completed hybrid systems, we now introduce the notion of a completed execution.

Definition 5: Given a simple hybrid Lagrangian $\mathbf{L}$ and the associated completed system $\overline{\mathrm{H}}_{\mathbf{L}}$, a completed execution $\bar{\chi}$ of $\overline{\mathrm{H}}_{\mathbf{L}}$ is a (possibly infinite) sequence of alternating hybrid and constrained executions $\bar{\chi}=\left\{\chi^{(1)}, \tilde{\chi}^{(2)}, \chi^{(3)}, \tilde{\chi}^{(4)}, \ldots\right\}$ that satisfies the following conditions.

(i) For each pair $\chi^{(i)}$ and $\tilde{\chi}^{(i+1)}$

$$
\tau_{\infty}^{(i)}=\tilde{\tau}_{0}^{(i+1)} \quad \text { and } \quad c_{\infty}^{(i)}=\tilde{c}_{0}^{(i+1)}\left(\tilde{\tau}_{0}^{(i+1)}\right) .
$$

(ii) For each pair $\tilde{\chi}^{(i)}$ and $\chi^{(i+1)}$

$$
\tilde{\tau}_{f}^{(i)}=\tau_{0}^{(i+1)} \text { and } \quad \tilde{c}^{(i)}\left(\tilde{\tau}_{f}^{(i)}\right)=c_{0}^{(i+1)}\left(\tau_{0}^{(i+1)}\right) .
$$

where the superscript $(i)$ denotes values corresponding to the $i$-th execution in $\bar{\chi}$, and $\tau_{\infty}^{(i)}, c_{\infty}^{(i)}$ denote the Zeno time 
and Zeno point associated with the $i$-th hybrid execution $\chi^{(i)}$.

Note that the first element of $\bar{\chi}$ can also be a constrained execution $\tilde{\chi}^{(1)}$, as long as the overall initial conditions satisfy conditions (i)-(iii) in (22). Note, too, that $\bar{\chi}$ can also consist of a finite number $N$ of executions, where the last execution (which can be either a constrained execution or a non-Zeno hybrid execution) extends to infinite time.

Remarks: First, as an interpretation of the completed execution, note that the only way to transition from a hybrid execution to a constrained execution is reaching a Zeno equilibrium point. The converse transition from a constrained execution to a hybrid execution occurs when the constraint force $\lambda$ crosses zero. Second, recall the expression for the acceleration $\ddot{h}(q, \dot{q})$ of $h(q(t))$ along trajectories of the unconstrained dynamics, which is given in (5). The definitions of $\ddot{h}(q, \dot{q})$ in (5) and $\lambda(q, \dot{q})$ in (21) imply that these two quantities are in complementarity relations, that is, while the solution slides along the surface $h^{-1}(0)$, either $\ddot{h}=0$ and $\lambda>0$, corresponding to maintaining constrained motion, or $\ddot{h}>0$ and $\lambda=0$, corresponding to leaving the constraint surface and switching back to the hybrid system. Thus, the definition of completed executions is consistent. Finally, note that the reason for considering both hybrid and constrained executions instead of directly defining the solution of the overall system $c(t)$ is that each hybrid execution has an infinite indexing set (the natural numbers). Therefore, using this definition, it would not be possible to consider a solution with more than one Zeno transition, since the impact times cannot be indexed. Thus the correct way to study these solutions is by considering them as a concatenation of multiple executions.

\section{PRactical Completion of Hybrid Systems}

In this section, we first discuss some practical difficulties arising in numerical simulation of completed hybrid systems near Zeno points, which inevitably lead to numerical errors. Motivated by these difficulties, we propose a procedure for computing a reliable approximation for the execution of a completed hybrid system, with guaranteed bounds on the approximation error.

\section{A. Practical Difficulties in Simulating Completed Hybrid Systems}

An important observation is that the notion of completed hybrid system described in Section IV is not practically useful; to be successfully implemented, one would need exact knowledge of Zeno executions, which cannot be assumed as it requires computing an infinite number of discrete transitions. Thus, one must be prepared to present a method for completing hybrid systems that will be amenable to simulation. That is, any software implementation of the numerical simulation of Zeno executions will necessarily involve a finite truncation of the infinite sequence of discrete transitions. Therefore, a notion of completing hybrid systems practically must be introduced-one that can handle errors introduced trough the finite truncation of Zeno executions.

Two main problems related to the reliability of these numerical approximations must be addressed. Firstly, one needs to guarantee that an approximation of a Zeno execution actually detects a true Zeno behavior of the exact execution and not just an aliasing effect resulting from truncations. Secondly, in the numerical simulation, the finite truncation is followed by choosing an approximate Zeno point, which then serves as an initial condition to the next phase of constrained dynamics. Therefore, in order to generate a reliable simulation, one needs guarantee that the approximated Zeno point lies in an arbitrarily close neighborhood of the exact Zeno limit point (which cannot be computed analytically). That is, the approximation error should satisfy a pre-specified bound. These two problems will be addressed through the utilization of results from Section III regarding sufficient conditions for stability of Zeno equilibria.

\section{B. Reliable Approximation of Completed Executions}

We now present the procedure for computing a reliable approximation for the execution of a completed hybrid system, with guaranteed bounds on the approximation error. The outline of the reliable approximation algorithm is as follows. First, a hybrid execution is simulated, until it reaches an impact at some time $\tau_{k}$, with the state $\left(q\left(\tau_{k}\right), \dot{q}\left(\tau_{k}\right)\right)$ satisfying certain conditions, called the reliable truncation conditions. At that point, the hybrid execution is truncated, and the algorithm applies a re-initialization map $R^{*}$ that maps the state $(q, \dot{q})$ at the time of truncation into an approximate Zeno equilibrium point $\left(q^{*}, \dot{q}^{*}\right) \in$ $Z$. The algorithm then switches to simulating the constrained dynamics (20), with the initial conditions given by $\left(q^{*}, \dot{q}^{*}\right)$.

We now define the re-initialization map $R^{*}$, and then define the reliable truncation conditions. The re-initialization map $R^{*}$ : $D_{\mathbf{L}} \rightarrow Z$ is given by $R^{*}(q, \dot{q})=\left(q^{*}, \dot{q}^{*}\right)$, where

$$
q^{*}=q \quad \text { and } \quad \dot{q}^{*}=\dot{q}-\frac{d h(q) \dot{q}}{\|d h(q)\|^{2}} d h(q)^{T} .
$$

Note that since $R^{*}$ is applied at an impact time, $q^{*}=q$ satisfies $h\left(q^{*}\right)=0$. Moreover, under the map $R^{*}$, the velocity $\dot{q}$ is projected orthogonally onto the plane $d h(q) \dot{q}=0$. Thus, it is clear that $\left(q^{*}, \dot{q}^{*}\right)$ is actually a Zeno equilibrium point.

We now define the reliable truncation conditions, depending on the given bounds $\epsilon_{q}, \epsilon_{v}$, and $\epsilon_{t}$, which are the desired bounds on the errors in position, velocity, and time, respectively, caused by the truncation.

Definition 6: Let $(q, \dot{q})$ be a state of $\mathrm{H}_{\mathbf{L}}$ such that $h(q)=$ 0 and $d h(q) \dot{q}<0$, and denote $\left(q^{*}, \dot{q}^{*}\right)=R^{*}(q, \dot{q})$. For given $\epsilon_{q}, \epsilon_{v}$, and $\epsilon_{t}$, define a neighborhood $V=N\left(\epsilon_{q}^{\prime}, \epsilon_{v}^{\prime}\right)$ of $\left(q^{*}, \dot{q}^{*}\right)$, that satisfies the conditions (6). The reliable truncation conditions for $(q, \dot{q})$ are then given by

(i) $\ddot{h}\left(q^{*}, \dot{q}^{*}\right)<0$

(ii) $|d h(q) \dot{q}|<\min \left\{\bar{\kappa}_{1}, \bar{\kappa}_{2}, \bar{\kappa}_{3}\right\}$

$$
\begin{aligned}
& \bar{\kappa}_{1}=\frac{a_{\min }(1-e \gamma)}{2 e} \epsilon_{t} \\
& \bar{\kappa}_{2}=\frac{a_{\min }(1-e \gamma)}{2 e \beta} \epsilon_{q}^{\prime} \\
& \bar{\kappa}_{3}=\frac{\epsilon_{v}^{\prime}}{\left(\frac{(1+e) \eta}{1-e \gamma}+\frac{2 e \zeta}{a_{\min }(1-e \gamma)}+\frac{1}{\left\|d h\left(q^{*}\right)\right\|}\right)}
\end{aligned}
$$


$\gamma, \epsilon_{q}^{\prime}, \epsilon_{v}^{\prime}, a_{\min }$ and $a_{\max }$ satisfy (6) and $e^{\prime}, \beta, \eta$ and $\zeta$ are defined in (10).

Note that the first condition in (23) is precisely the condition for local stability of the Zeno equilibrium point $\left(q^{*}, \dot{q}^{*}\right)$. The second condition in (23) requires that the pre-impact velocity at the truncation time $\dot{h}\left(q\left(\tau_{k}\right)\right)$ is sufficiently small. The following theorem states that the reliable truncation conditions guarantee the desired bounds on the error between the exact Zeno point $\left(q_{\infty}, \dot{q}_{\infty}\right)$ and the truncated and re-initialized state $\left(q^{*}, \dot{q}^{*}\right)$, as well as on the exact Zeno time. This result stems directly from Theorem 2 and Lemmas 1, 2, and 3 in Section III.

Theorem 3: Let $\chi^{\mathrm{H}_{\mathrm{L}}}$ be an execution of a simple Lagrangian hybrid system $\mathrm{H}_{\mathbf{L}}$. Then if there exists $k \in \Lambda$ such that $\left(q_{k-1}\left(\tau_{k}\right), \dot{q}_{k-1}\left(\tau_{k}\right)\right)$ satisfy the reliable truncation conditions with respect to given $\epsilon_{q}, \epsilon_{v}$ and $\epsilon_{t}$, then $\chi^{\mathrm{H}_{\mathrm{L}}}$ is a Zeno execution, whose Zeno time satisfies $\tau_{\infty}-\tau_{k}<\epsilon_{t}$, and its Zeno point satisfies $\left\|q_{\infty}-q^{*}\right\|<\epsilon_{q}$ and $\left\|\dot{q}_{\infty}-\dot{q}^{*}\right\|<\epsilon_{v}$, where $\left(q^{*}, \dot{q}^{*}\right)=R^{*}\left(q_{k-1}\left(\tau_{k}\right), \dot{q}_{k-1}\left(\tau_{k}\right)\right)$.

Proof: The proof utilizes the construction of the neighborhood $W^{\prime}$ in (11), and the results of Lemma 2 from Section III. First, note that condition (i) in (23) guarantees that $\left(q^{*}, \dot{q}^{*}\right)$ satisfies the BTLS conditions, and that the neighborhood $V=N\left(\epsilon_{q}^{\prime}, \epsilon_{v}^{\prime}\right)$ exists. Choosing $\delta_{q}^{\prime}=0$ and $\delta_{v}^{\prime}=d h\left(q^{*}\right) \dot{q} /\left\|d h\left(q^{*}\right)\right\|$, and defining the neighborhood $W^{\prime}$ of $\left(q^{*}, \dot{q}^{*}\right)$ as in (11), the definition of $R^{*}$, along with condition (ii) in (23) imply that $\left(q_{k-1}\left(\tau_{k}\right), \dot{q}_{k-1}\left(\tau_{k}\right)\right) \in W^{\prime}$. We can then exploit the time-invariance of $\mathbf{H}_{\mathbf{L}}$ to shift the time $\tau_{k}$ to $\tau_{1}$, and treat the remaining part of the execution $\chi^{\mathrm{H}_{\mathbf{L}}}$ of times $t \geq \tau_{k}$ (i.e., past the truncation point) as an execution in the vicinity of $\left(q^{*}, \dot{q}^{*}\right)$, having initial conditions within $W^{\prime}$. Lemma 2 then implies the desired bounds on the Zeno point and the Zeno time of $\chi^{\mathrm{H}_{\mathrm{L}}}$, where the definitions (24) are straightforward substitution of the chosen $\delta_{q}^{\prime}$ and $\delta_{v}^{\prime}$ into (12).

1) The Completion Procedure: We now summarize the procedure for practical simulation of a completed Lagrangian hybrid system $\overline{\mathrm{H}}_{\mathbf{L}}$ under desired bounds $\epsilon_{q}, \epsilon_{v}, \epsilon_{t}$ on the truncation errors.

1) Initialization: set an initial time $\tau_{0}$ and initial conditions $\left(q\left(\tau_{0}\right), \dot{q}\left(\tau_{0}\right)\right)$.

2) If $h\left(q\left(\tau_{0}\right)\right)=0$ and $d h\left(q\left(\tau_{0}\right)\right) \dot{q}\left(\tau_{0}\right)=0$, go to step 5 .

3) Simulate an execution of the hybrid system $H_{L}$, until it reaches an impact at time $\tau_{k}$ such that $\left(q_{k-1}\left(\tau_{k}\right), \quad \dot{q}_{k-1}\left(\tau_{k}\right)\right)$ satisfy the reliable truncation conditions.

4) Set $\tilde{\tau}_{0}=\tau_{k}$ and $\left(q\left(\tilde{\tau}_{0}\right), \dot{q}\left(\tilde{\tau}_{0}\right)\right)=R^{*}\left(q\left(\tau_{k}\right), \dot{q}\left(\tau_{k}\right)\right)$.

5) Simulate the constrained system $D_{\mathbf{L}}$ until reaching a time $\tilde{\tau}_{f}$ at which $\lambda\left(q\left(\tilde{\tau}_{f}\right), \dot{q}\left(\tilde{\tau}_{f}\right)\right)=0$.

6) Set $\tau_{0}=\tilde{\tau}_{f}$ and $\left(q\left(\tau_{0}\right), \dot{q}\left(\tau_{0}\right)\right)=\left(q\left(\tilde{\tau}_{f}\right), \dot{q}\left(\tilde{\tau}_{f}\right)\right)$.

7) Return to step 3.

Remarks: It is important to discuss the ramifications of the proposed procedure, which will be done through a series of remarks. First, note that in some cases, step 3 or step 5 may never terminate. This happens when either the hybrid execution is not Zeno and extends to infinite time, or when the constrained execution satisfies $\lambda>0$ for infinite time. Second, note that in practice the quantities $a_{\min }, a_{\max }, \eta, \beta$ and $\zeta$ need not be computed exactly. Instead, one can use simplified conservative approximations of them, e.g., $\tilde{a}_{\min }<a_{\min }, \tilde{a}_{\max }>a_{\max }, \tilde{\beta}>\beta$, et cetera. Third, note that Theorem 3 only implies that the bounds on the truncation error hold for a single truncation, and not for the overall cumulative error of a long-time simulation with multiple truncations. Other possible sources of numerical errors in simulation of hybrid executions are numerical integration errors during the continuous phases of constrained and unconstrained motion, as well as inaccuracies in the detection of zero-crossing events $h(q(t))=0$ and in the re-initialized state. While discussion of numerical integration errors is beyond the scope of this paper, the problem of event detection and re-initialization inaccuracies can be partially solved by defining an alternative set of coordinates $q^{\prime}$ such that $h(q)$ is one of the new coordinates. This enables easier detection of the event $h=0$, and allows for manually enforcing $h=0$ at any post-impact configuration $q^{\prime}$. Moreover, these coordinates are also useful for numerically integrating the constrained dynamics $\mathrm{D}_{\mathbf{L}}$ with higher accuracy, by enforcing $h=\dot{h}=0$ at each time step. Finally, note that the practical completion procedure described above essentially approximates a completed Lagrangian hybrid system by transforming it into a hybrid system with two domains, where the conditions of reliable truncation play the role of a (rather complicated) guard, and the re-initialization map $R^{*}$ is the reset map that sends the solution to the constraint surface $h^{-1}(0)$, which is the second domain. This approximation enables practical simulation and further numerical investigation of the completed hybrid system.

\section{Simulation Results}

In this section, we present numerical simulations for the examples considered in Section II.

1) Example 3 (Ball): Consider the example of a ball bouncing on a sinusoidal surface [Fig. 1(a)]. Recall that the set of Zeno equilibria for this system are states at which the ball slides along the sinusoidal surface. The stability of each Zeno equilibrium point is determined according to Theorem 2, and depends on the sign of $\ddot{h}(q, \dot{q})$. Using the definition in (5), the stability condition is given by $\ddot{h}(q, \dot{q})=v_{x}^{2} \sin (x)-g<0$, where we denote $\dot{q}=\left(v_{x}, v_{y}\right)$. This indicates that Zeno equilibrium points that satisfy $\sin (x)<0$ i.e., near the "valleys", are always stable, and are more likely to attract Zeno executions. On the other hand, points such that $\sin (x)>0$, i.e., on the "hills", can be made stable only by setting the horizontal velocity $v_{x}$ sufficiently small.

In our simulation, the values of system's parameters were chosen as $m=1, g=1$ and $e=0.5$. We simulated this system under two different sets of initial conditions, where in both cases the initial conditions at $t=0$ are chosen such that at $\tau_{1}=0.05$, a first impact occurs at $x\left(\tau_{1}\right)=0.3, y\left(\tau_{1}\right)=\sin (0.3)$. In the first case, the initial velocities are chosen as $v_{x}(0)=1.8$ and $v_{y}(0)=0$. The execution was simulated until an impact time $\tau_{k}$ at which the pre-impact velocity $d h\left(q_{k-1}\left(\tau_{k}\right)\right) \dot{q}_{k-1}\left(\tau_{k}\right)$ is less than $10^{-10}$. The results of this simulation are shown in Fig. 4, as follows. Figs. 4(a)-4(e) show the time plots of $x(t), y(t), v_{x}(t), v_{y}(t)$ and $h(q(t))$, respectively. The points of impact events are marked with squares (" $\square$ "). Fig. 4(f) plots $x(t)$ versus $y(t)$, with the constraint surface $y=\sin (x)$ appearing as a pale (green) solid curve. This simulation results in a Zeno execution that converges at a Zeno time $\tau_{\infty}=3.761$ 


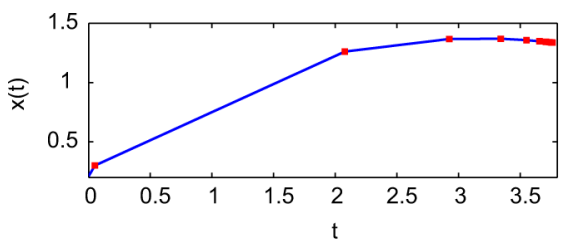

(a)

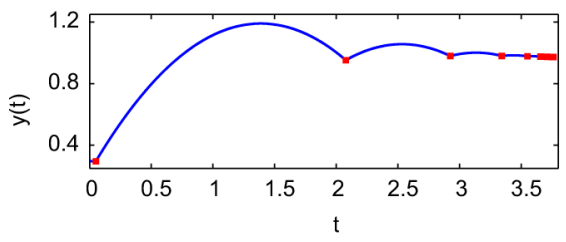

(b)

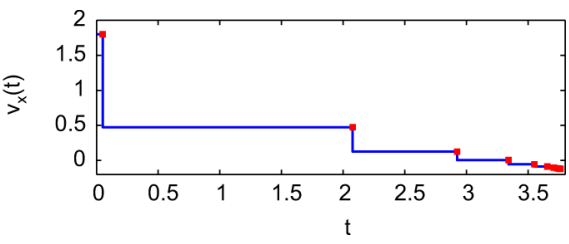

(c)

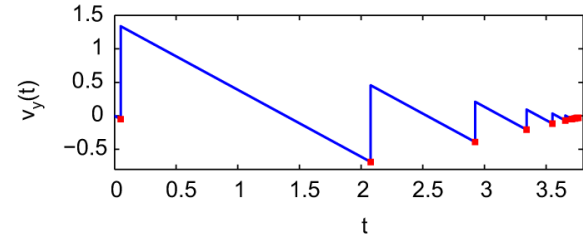

(d)

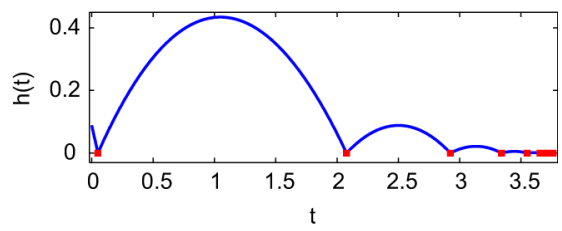

(e)

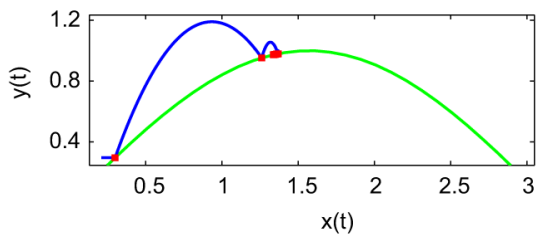

(f)

Fig. 4. Simulation results for the ball example with initial velocities $v_{x}(0)=1.8$ and $v_{y}(0)=0$.

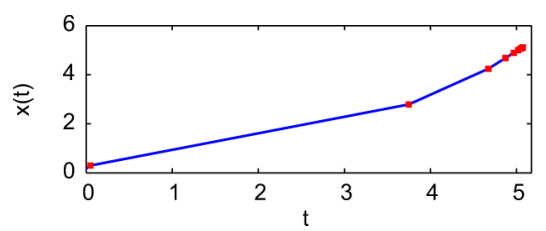

(a)

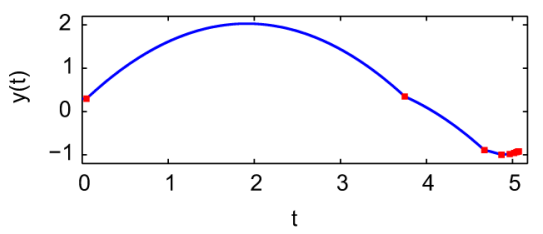

(b)

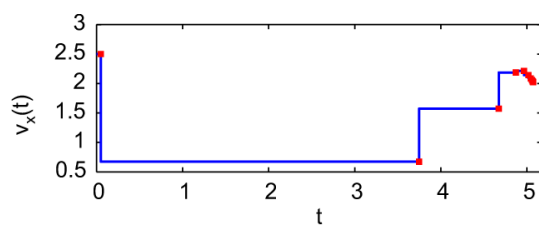

(c)

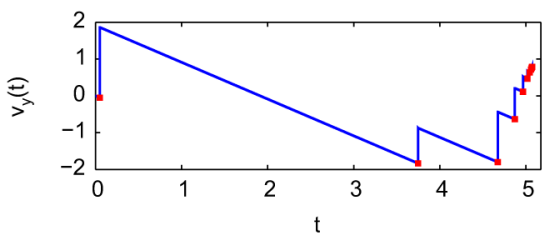

(d)

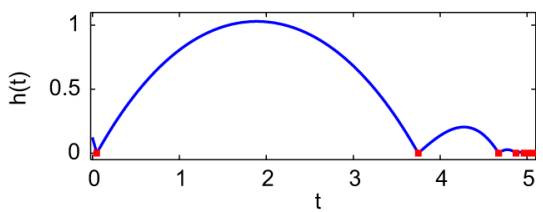

(e)

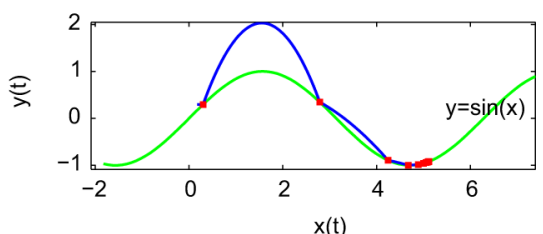

(f)

Fig. 5. Simulation results for the ball example with initial velocities $v_{x}(0)=2.5$ and $v_{y}(0)=0$.

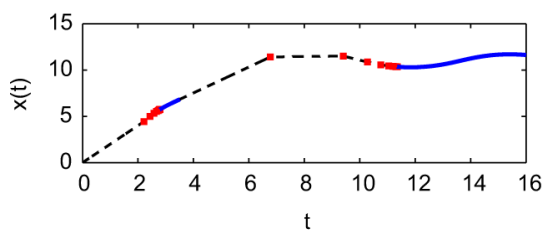

(a)

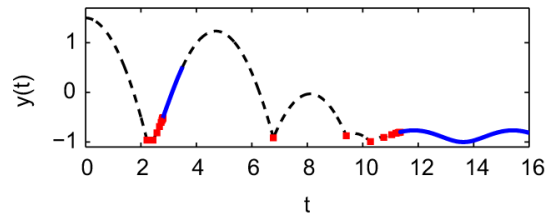

(b)

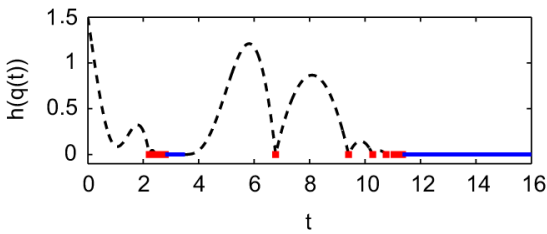

(c)

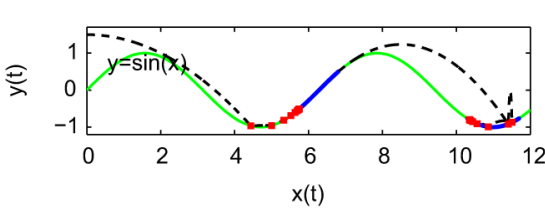

(d)

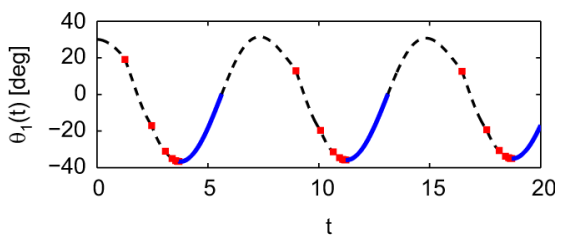

(e)

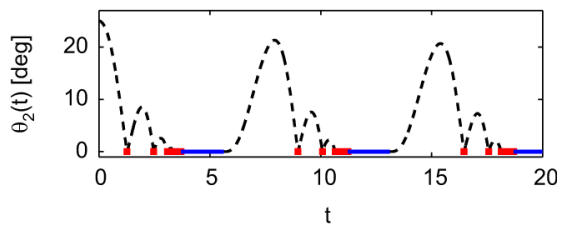

(f)

Fig. 6. Simulation results for: (a)-(d) the ball on a sinusoidal surface and (e)-(f) the double pendulum with a mechanical stop.

to the Zeno equilibrium point $q^{*}=(1.337,0.973)$ and $\dot{q}^{*}=$ $(-0.121,-0.028)$. This Zeno point is close to a maximum point of the surface; note that the horizontal velocity $v_{x}$ is significantly decreased from its initial value, so that $\ddot{h}\left(q^{*}, \ddot{q}^{*}\right)=-0.986<0$ and the stability condition is satisfied. Note, too, that the behavior of $h(q(t))$ in the vicinity of the Zeno point (Fig. 4(e)) is remarkably similar to that of a simple bouncing ball.

In the second case, the initial velocities are chosen as $v_{x}(0)=$ 2.5 and $v_{y}(0)=0$. Figs. 5(a) $-5(\mathrm{f})$ show the simulation results under these initial conditions. This simulation results in a Zeno execution that converges at a Zeno time $\tau_{\infty}=5.0731$ to the Zeno equilibrium point $q^{*}=(5.114,-0.920)$ and $\dot{q}^{*}=$ $(2.023,0.791)$. One can see that the trajectory is initially "repelled" from the maximum point due to the large horizontal velocity, and attracted towards the next minimum point, while the horizontal velocity is increased, such that $\ddot{h}\left(q^{*}, \ddot{q}^{*}\right)=-4.766$ satisfies the stability condition in Theorem 2 .

Next, we move beyond the Zeno time, by simulating the completed hybrid system for this example. Figs. 6(a)-6(c) show time plots of $x(t), y(t)$ and $h(q(t))$, respectively, under initial con- 
ditions $q(0)=(0,2)$ and $\dot{q}(0)=(1.5,0)$. Solutions of constrained executions appear as solid curves, while solutions of hybrid executions appear as dashed curves. The points of impact events are marked with squares (“ø"). Fig. 6(d) plots $x(t)$ versus $y(t)$, with the constraint surface $y=\sin (x)$ appearing as pale (green) solid line. The results of this representative simulation show initial bouncing of the ball which converges to a Zeno equilibrium point at approximately $t=3$. Then the system switches to a constrained motion until the contact force $\lambda(q, \dot{q})$ vanishes at approximately $t=4$. The system then switches again to a hybrid execution, which converges to a Zeno point at approximately $t=11$. Finally, switching again to the constrained dynamics, the solution is then "trapped" near a minimum, and exhibits an undamped pendulum-like periodic motion for infinite time (since the constrained dynamics (20) does not include any dissipation terms).

2) Example 4 (Double Pendulum): In the second running example (Example 2) consisting of a double pendulum with a mechanical stop [Fig. 1(b)], the condition for stability of Zeno equilibria given in Theorem 2 is $\ddot{h}(q, \dot{q})=g \sin \theta_{1} / \tilde{L}<0$, where $\tilde{L}=\left(4 m_{1}+3 m_{2}\right) L_{1} L_{2} / 3\left(m_{1}\left(L_{1}+2 L_{2}\right) m_{2} L_{2}\right)$. This indicates that only points at which $\sin \theta_{1}<0$ (i.e., the links are inclined to the left) are stable Zeno equilibria. We now show simulations of the completed hybrid systems for this example, where we chose the numerical parameters $m_{1}=m_{2}=L_{1}=$ $L_{2}=g=1$, and a coefficient of restitution $e=0.5$. Figs. 6(e) and 6(f) show the time plots of $\theta_{1}(t)$ and $\theta_{2}(t)$ under initial conditions $q(0)=\left(30^{\circ}, 25^{\circ}\right)$ and $\dot{q}(0)=(0,0)$. Again, solutions of constrained executions appear as solid curves, while solutions of hybrid executions appear as dashed curves. The results show a seemingly periodic motion, at which link 2 repeatedly hits the mechanical stop and bounces, until it converges to a Zeno point at which $\theta_{1}<0$ and establishes contact. Then the two links attach and swing rigidly as a single pendulum in a constrained motion. When $\theta_{1}$ crosses zero, the constraining force $\lambda$ vanishes and the two links separate again, as predicted by the stability condition. At this point the dynamics switches back to the hybrid system, and the solution exhibits a sequence of impact events and converges again to a Zeno equilibrium point at $\theta_{1} \approx-40^{\circ}$. One can notice that this periodic-like motion is actually decaying, due to the energy dissipation induced by the impacts. Exact periodic orbits with Zeno behavior in a controlled version of this system are analyzed in [31].

\section{DisCUSSION-STABILITY OF CONSTRAINED MECHANICAL SYSTEMS}

In this section we discuss a key interpretation of the results in this paper to stability of mechanical systems with unilateral contacts. Typical examples of such systems are robotic manipulation [19], and bipedal locomotion [46]. An important open problem for these systems is analysis of their behavior when the constrained motion undergoes small position-and-velocity perturbations that violate the contact constraints. Such perturbations may originate from external disturbances or small errors in the coordinated motion of internal links, causing instantaneous contact separation. This problem has not been previously addressed in the literature, primarily due to lack of a coherent framework for treating transitions between hybrid dy- namics and constrained dynamics. For example, works that analyze the stability of robotic grasps [19] and bipedal locomotion [46] consider only stability with respect to the subset of perturbations under which the contact constraints are maintained. Other works verify the consistency of the constrained motion by guaranteeing that the contact forces are nonnegative at all times, such as [48] in the context of manipulation, and [11], [45] which employ the ZMP criterion for bipedal locomotion. However, none of these works analyze stability under perturbations that violate the contact constraint. An exception is the work by Or and Rimon [33], which analyzes the stability of an equilibrium posture of a planar rigid body supported by two unilateral contacts with friction. Although this work focuses on a specific case, it is the motivation for the present discussion.

The constrained dynamics of a mechanical system with a single unilateral constraint is formulated in (20). When the constraint $h(q(t))=0$ is satisfied for some time interval the state $(q, \dot{q})$ of this system is restricted to the constraint surface $\mathcal{C}$, defined by $\mathcal{C}=\{(q, \dot{q}) \in T Q: h(q)=0$ and $d h(q) \dot{q}=0\}$. When allowing initial perturbations that violate the constraint, one needs to consider executions of the associated completed hybrid system, as described in Section IV. We now utilize the framework of completed Lagrangian hybrid system proposed in this paper to define the new notion of constraint stability, as follows:

Definition 7: Consider a completed Lagrangian hybrid system $\overline{\mathrm{H}}_{\mathrm{L}}$ associated with the Lagrangian $L(q, \dot{q})$ and the constraint $h(q) \geq 0$, and let $x^{\prime}=\left(q^{\prime}, \dot{q}^{\prime}\right)$ be a point in $\mathcal{C}$. Then $\overline{\mathrm{H}}_{\mathbf{L}}$ possesses constraint stability at $x^{\prime}$ if for each open neighborhood $U \subseteq T Q$ of $x^{\prime}$, there exists another open neighborhood $W$ of $x^{\prime}$, such that for every initial condition $c_{0}\left(\tau_{0}\right) \in W \cap D_{\mathbf{L}} \backslash \mathcal{C}$, the corresponding hybrid execution $\chi^{(1)}$ is a Zeno execution that satisfies $c_{i}^{(1)}(t) \in U$ for all $t \in I_{i}^{(1)}$ and $i \in \Lambda^{(1)}$, and converges to a Zeno point $x_{\infty}=\left(q_{\infty}, \dot{q}_{\infty}\right) \in \mathcal{C}$ that satisfies $\lambda\left(q_{\infty}, \dot{q}_{\infty}\right)>0$.

The interpretation of this definition is that constraint stability at $x^{\prime} \in D$ simply requires that under any small initial perturbation about $x^{\prime}$ that violates the constraint, the dynamic response of the system converges back to the constraint surface via a Zeno execution while staying within a small neighborhood of $x^{\prime}$. The following corollary is a straightforward implication of the results presented in previous sections.

Corollary 1: Let $\overline{\mathrm{H}}_{\mathbf{L}}$ be a Lagrangian hybrid system associated with the Lagrangian $L(q, \dot{q})$ and the constraint $h(q) \geq 0$, and assume that $e<1$. Let $x^{\prime}=\left(q^{\prime}, \dot{q}^{\prime}\right)$ be a point in $\mathcal{C}$. Then if $\lambda\left(q^{\prime}, \dot{q}^{\prime}\right)>0$, then $\overline{\mathrm{H}}_{\mathbf{L}}$ possesses constraint stability at $x^{\prime}$, where the expression for $\lambda(\cdot, \cdot)$ is given in (21).

Proof: First, note that $x^{\prime} \in \mathcal{C}$ is a Zeno equilibrium point of the hybrid system $\mathrm{H}_{\mathbf{L}}$. Second, note that bounded-time local stability of $x^{\prime}$ (Definition 4) implies constraint stability of $\overline{\mathrm{H}}_{\mathbf{L}}$ at $x^{\prime}$ (Definition 7). Finally, (5) and (21) imply that if $\lambda\left(q^{\prime}, \dot{q}^{\prime}\right)>0$ then $\ddot{h}\left(q^{\prime}, \dot{q}^{\prime}\right)<0$, which, according to Theorem 2 , guarantees the bounded-time local stability of $x^{\prime}$.

The physical interpretation of this result is that if the constraint force $\lambda$ is strictly positive along solutions of the constrained dynamics, then the system's response under small position-and-velocity perturbations that violate the constraint is guaranteed to converge back to the constraint surface. Although the result is 
highly intuitive, the authors are unaware of any similar result in the literature. In some sense, it provides a partial justification to previous works that use the term "stability test" to a simple check of the positivity of the constraint force required to maintain contact. However, one must keep in mind that Theorem 1 is currently limited to mechanical systems with a single unilateral constraint, involving frictionless constrained dynamics of the form (20), under frictionless impacts of the form (4). The extension of this result to frictional contact and to higher number of unilateral constraints introduces additional difficulties, which are briefly discussed in the concluding section.

\section{CONCLUSION}

In this paper, we studied Zeno behavior in Lagrangian hybrid systems and derived necessary and sufficient conditions for stability of Zeno equilibria. We have derived explicit bounds on the neighborhood of initial conditions that guarantee convergence of the solution to a Zeno equilibrium point in an arbitrarily small Zeno time, while staying bounded within arbitrarily small neighborhood. Then we have formally defined the notion of completed hybrid systems, under which solutions are extended beyond the Zeno time by two-way transitions between the hybrid dynamics and the holonomically constrained dynamics. Additionally, we presented a procedure for practical simulation of completed hybrid systems, and derived conditions under which a Zeno execution can be reliably truncated after a finite number of transitions while guaranteeing that the resulting numerical error is less than a pre-specified bound. Finally, we have discussed the interpretation of the results to stability of unilaterally constrained motion of mechanical systems under perturbations that violate the constraint.

We now briefly discuss limitations of the results and list some open problems for future research. First, note that the results are currently limited to mechanical systems with a single unilateral constraints. Generalization to systems with multiple constraints is challenging, both in increasing the complexity of the system's structure due to existence of multiple domains, and in correct modeling of impact at multiple contacts [25]. Second, the results are currently limited to frictionless contacts. Extension to frictional contact models poses problems of solution inconsistency and indeterminacy [8], [36]. In a particular case, Or and Rimon analyze the hybrid dynamics and stability of equilibrium postures for a rigid body supported by two frictional contacts [32], [33]. However, this preliminary result is still far from presenting a complete framework for treating the general case of multiple frictional contacts. Finally, the paper focuses only on Lagrangian hybrid system. Extensions of the results to more general classes of hybrid systems, such as set-valued systems, is still under investigation [15], [34].

\section{APPENDIX}

In this Appendix, we prove Lemma 1 and a result from Lemma 3 by utilizing methods from optimal control, namely, Pontryagin's maximum principle. (The idea of using optimal control to analyze stability of differential inclusions also appears in the work of Liberzon and Margaliot [24].) We, therefore, briefly review the basic form of this principle based on its presentation in [6], though we adopt a slightly different notation.
Consider a control system

$$
\dot{x}=f(x, u)
$$

where $x \in \mathbb{R}^{n}$ and $u \in \Omega \subseteq \mathbb{R}^{m}$, and $\Omega$ is a convex set of admissible controls. A solution to (25) on a time interval $\left[t_{0}, t_{f}\right]$ is a pair $(x(t), u(t))$ satisfying (25) and $u(t) \in \Omega$ for all $t \in$ $\left[t_{0}, t_{f}\right]$; the initial and final conditions of $x(t)$ are denoted $x_{0}=$ $x\left(t_{0}\right)$ and $x_{f}=x\left(t_{f}\right)$. The design goal is to find a solution to (25) that minimizes a given cost function $P\left(x_{f}, t_{f}\right)^{1}$. The end condition $x_{f}$ and the end time $t_{f}$ can either be specified, or left as free parameters of optimization.

Using calculus of variations techniques, the solution of this problem is given as follows. First, define the Hamiltonian, given by $H(x, u, \lambda, t)=\lambda(t)^{T} f(x, u)$, where $\lambda \in \mathbb{R}^{n}$ is called the co-state vector. The co-state dynamic equations are then given by $\dot{\lambda}=-\partial H / \partial x$, and the optimal control satisfies $u^{*}(t)=\arg \min H$. The end condition is given by $\left[\partial P / \partial x_{f}-\lambda\left(t_{f}\right)\right]^{T} \delta x_{f}=0$, where if a particular state variable $x_{i}$ is specified, then its variation $\delta x_{i}\left(t_{f}\right)$ vanishes, and if it is not specified, then it gives an end condition for the corresponding co-state variable $\lambda_{i}$. In case where the terminal time $t_{f}$ is not specified, an additional condition on $H\left(t_{f}\right)$ is given by $\partial P / \partial t_{f}+H\left(t_{f}\right)=0$.

Proof of Lemma 1: The main idea of the proof is as follows. Recall that assuming $c_{i}(t) \in V$ for all $t \in I_{i}$ and $i \in\{1 \ldots k\}$ implies that the constraint function $h(q(t))$ satisfies the differential inclusion $\ddot{h}(t) \in\left[-a_{\max },-a_{\text {min }}\right]$ for all times $t \in I_{i}$ and $i \in$ $\{1 \ldots k\}$. The impact law (4) implies the relation $v_{i}^{+}=-e v_{i}^{-}$ where $v_{i}^{-}$and $v_{i}^{+}$are the left and right limits, respectively of the velocity $\dot{h}(t)$ at the impact times $\tau_{i}$. This set-valued hybrid system describes a "set-valued bouncing ball" system [34]. Our goal is to find bounds on all possible solution on this system, which will necessarily hold also for $h(q(t))$ along the unknown solution of the original system. As the first step, choosing a state vector $x=\left(x_{1}, x_{2}\right)=(h(q), \dot{h}(q, \dot{q}))$, the differential inclusion can be reformulated as a control system

$$
\left\{\begin{array}{l}
\dot{x}_{1}=x_{2} \\
\dot{x}_{2}=u
\end{array} \quad \text { where } \quad u \in\left[-a_{\max },-a_{\min }\right] .\right.
$$

The start and end times $t_{0}$ and $t_{f}$ represent two consecutive impact times $\tau_{i}$ and $\tau_{i+1}$. The initial conditions are thus $x_{1}\left(t_{0}\right)=0$ and $x_{2}\left(t_{0}\right)=v_{i}^{+}>0$. Since $\dot{x}_{2}$ in (26) is always strictly negative, there must exist a time $t_{f}>0$ for which $x_{1}\left(t_{f}\right)=0$ and $x_{2}\left(t_{f}\right)<0$, corresponding to the next impact event. Using $t_{f}$ as the terminal time, one end condition is specified, namely $x_{1}\left(t_{f}\right)=0$, while the terminal velocity $x_{2}\left(t_{f}\right)=v_{i+1}^{-}$, as well as the terminal time itself, are both unspecified and left as free parameters for optimization.

In order to prove (8), consider the cost function: $P\left(x_{f}, t_{f}\right)=$ $x_{2}\left(t_{f}\right)$ for the control system (26). The Hamiltonian is given by $H=\lambda_{1} x_{2}+\lambda_{2} u$. The co-state dynamic equations are then $\dot{\lambda}_{1}=$ 0 and $\dot{\lambda}_{2}=-\lambda_{1}$, indicating that $\lambda_{1}(t)$ is constant and $\lambda_{2}(t)$ is a linear function. The end condition gives $\lambda_{2}\left(t_{f}\right)=1$. The maximum principle then implies that the optimal input $u^{*}(t)$ is

\footnotetext{
${ }^{1}$ Many textbooks also consider an integral cost function of the form $J=$ $\int_{t_{0}}^{t} g(x, u, t) d t$. This cost function can be incorporated into the formulation here by using an additional state variable $z$, whose dynamics is given by $\dot{z}=$ $g(x, u, t)$. The cost function is then simply given by $P=z\left(t_{f}\right)$.
} 
either $a_{\min }$ or $a_{\max }$, and depends solely on the sign of $\lambda_{2}(t)$, which is a linear function that has at most one zero-crossing point. Therefore, $u^{*}$ is a piecewise-constant function with at most one switching time, hence we can set $u^{*}(t)=-u_{1}$ for $t \in\left[t_{0}, t_{s}\right]$ and $u^{*}(t)=-u_{2}$ for $t \in\left[t_{s}, t_{f}\right]$, where $t_{s}$ is the switching time, and $u_{1}, u_{2} \in\left\{-a_{\max },-a_{\min }\right\}$.

Substituting the expression for $u^{*}(t)$, direct integration of (12) with the given initial conditions yields the solution for the terminal velocity as $x_{2}\left(t_{f}\right)=-\sqrt{\left(v_{i}^{+}-u_{1} t_{s}\right)^{2}+2 u_{2}\left(v_{i}^{+} t_{s}-u_{1} t_{s}^{2} / 2\right)}$. Differentiating with respect to $t_{s}$, it is straightforward to show that $x_{2}\left(t_{f}\right)$ attains a critical value for the switching time $t_{s}^{*}=v_{i}^{+} / u_{1}$. Direct substitution then shows that the maximal value of $\left|x_{2}\left(t_{f}\right)\right|$ is obtained by taking $u_{1}=-a_{\min }$ and $u_{2}=-a_{\max }$, and is given by $x_{2}^{*}\left(t_{f}\right)=-\sqrt{a_{\max } / a_{\min }} v_{i}^{+}$. Note that the optimal solution of $x_{2}^{*}(t)$ vanishes at the switching time $t_{s}^{*}$. The physical meaning of the optimal solution is that for the set-valued bouncing ball described in (26), maximum hitting velocity is attained by choosing the "slowest" acceleration $\ddot{h}(t)=-a_{\min }$ for the way up, i.e., $\dot{h}(t)>0$, and the "fastest" acceleration $\ddot{h}(t)=-a_{\max }$ for the way down, i.e., $\dot{h}>0$. Augmenting the result with the relation $v_{i}^{+}=-e v_{i}^{-}$, the sequence of pre-impact velocities satisfies the bound $\left|v_{i+1}^{-}\right| \leq e \gamma\left|v_{i+1}^{-}\right|$. Using similar arguments, one can find the minimum of $\left|x_{2}\left(t_{f}\right)\right|$, and obtain the lower bound $\left|v_{i+1}^{-}\right| \geq e / \gamma\left|v_{i+1}^{-}\right|$, which completes the proof of (8).

In order to prove (9), consider again the control system (26) with the cost function $P\left(x_{f}, t_{f}\right)=t_{f}$. Following similar derivation, the Hamiltonian is again given by $H=\lambda_{1} x_{2}+\lambda_{2} u$. The co-state dynamic equations are again $\dot{\lambda}_{1}=0$ and $\dot{\lambda}_{2}=-\lambda_{1}$, indicating that $\lambda_{1}(t)$ is constant and $\lambda_{1}(t)$ is a linear function. The end condition for $\lambda_{2}$ now gives $\lambda_{2}\left(t_{f}\right)=0$. The additional condition associated with the terminal time now gives $H\left(t_{f}\right)=-1$, which rules out the trivial solution $\lambda_{1}(t)=\lambda_{2}(t)=0$. Since $\lambda_{2}(t)$ crosses zero only at the terminal time $t=t_{f}$, the maximum principle implies that the optimal solution is obtained by taking constant input $u$ without switching, and it is simple to verify that taking $u=-a_{\min }$ gives the maximum time $\Delta \tau_{i}=2 v_{i}^{+} / a_{\min }$. Using the relation $v_{i}^{+}=-e v_{i}^{-}$then gives the bound (9).

Finally, in order to prove the bound (19) from Lemma 3, consider the optimal control problem (26), with initial condition $x\left(t_{0}\right)=\left(h_{0}, v_{0}\right)$ and end condition $x_{1}\left(t_{f}\right)=0$. The cost function to be minimized is again $P\left(x_{f}, t_{f}\right)=x_{2}\left(t_{f}\right)$, where the initial and final times $t_{0}$ and $t_{f}$ represent the times $\tau_{0}$ and $\tau_{1}$. Using similar derivation as above, it can be shown that the optimal solution is obtained by taking $u(t)=-a_{\min }$ when $x_{2}(t)>0$, and $u(t)=-a_{\min }$ when $x_{2}(t)>0$. Since $x_{2}(t)$ is monotonously decreasing under (26), if $v_{0} \geq 0$ we have that $u^{*}(t)=-a_{\max }$ at all times, while if $v_{0}<0, u^{*}(t)$ has a switch from $-a_{\min }$ to $a_{\max }$ at some time $t_{s}$. Following the same calculations as in the proof of (8), direct integration of (26) under the optimal control $u^{*}(t)$ gives

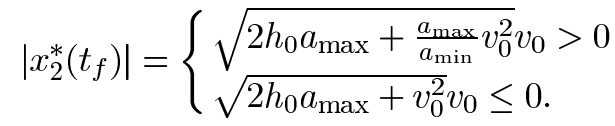

Interpreting $\left|x_{2}^{*}\left(t_{f}\right)\right|$ as the upper bound of $\left|v_{1}^{-}\right|$then completes the proof of (19).

\section{REFERENCES}

[1] A. D. Ames, A. Abate, and S. Sastry, "Sufficient conditions for the existence of Zeno behavior," in Proc. IEEE Conf. Decision and Control and European Control Conf., 2005, pp. 696-701.

[2] A. D. Ames, A. Abate, and S. Sastry, "Sufficient conditions for the existence of Zeno behavior in nonlinear hybrid systems via constant approximations," in Proc. IEEE Conf. Decision and Control, 2007, pp. 4033-4038.

[3] A. D. Ames and R. D. Gregg, "Stably extending two-dimensional bipedal walking to three dimensions," in Proc. American Control Conf., 2007, pp. 2848-2854.

[4] A. D. Ames, R. Sinnet, and E. Wendel, "Three-dimensional kneed bipedal walking: A hybrid geometric approach," in Hybrid Systems: Computation and Control, ser. Lecture Notes in Computer Science. New York: Springer-Verlag, 2009, vol. 5469, pp. 16-30.

[5] A. D. Ames, H. Zheng, R. D. Gregg, and S. Sastry, "Is there life after Zeno? taking executions past the breaking (Zeno) point," in Proc. American Control Conf., 2006, pp. 2652-2657.

[6] M. Athans and P. L. Falb, Optimal Control: An Introduction to the Theory and Its Applications. New York: McGraw-Hill, 1966.

[7] J. Bourgeot and B. Brogliato, "Tracking control of complementarity Lagrangian systems," Int. J. Bifurc. and Chaos, vol. 15, no. 6, pp. $1839-1866,2005$.

[8] B. Brogliato, Nonsmooth Mechanics. New York: Springer-Verlag, 1999.

[9] M. K. Camlibel and J. M. Schumacher, "On the Zeno behavior of linear complementarity systems," in Proc. IEEE Conf. Decision and Control, 2001, pp. 346-351.

[10] A. Chatterjee and A. Ruina, "A new algebraic rigid body collision law based on impulse space considerations," J. Appl. Mechan., vol. 65, no. 4, pp. 939-951, 1998.

[11] C. Chevallereau, D. Djoudi, and J. Grizzle, "Stable bipedal walking with foot rotation through direct regulation of the zero moment point," IEEE Trans. Robot. Autom., vol. 25, no. 2, pp. 390-401, Apr. 2008.

[12] C. Chevallereau, E. Westervelt, and J. Grizzle, "Asymptotically stable running for a five-link, four-actuator, planar bipedal robot," Int. J. Robot. Res., vol. 24, no. 6, pp. 431-464, 2005.

[13] A. Filippov, Differential Equations With Discontinuous Right-Hand Sides. Norwell, MA: Kluwer, 1988.

[14] R. Goebel, J. Hespanha, A. Teel, C. Cai, and R. Sanfelice, "Hybrid systems: Generalized solutions and robust stability," in Proc. 6th IFAC Symp. Nonlinear Control Systems, 2004, pp. 1-12.

[15] R. Goebel and A. R. Teel, "Lyapunov characterization of Zeno behavior in hybrid systems," in Proc. IEEE Conf. Decision and Control, 2008, pp. 2752-2757.

[16] J. Grizzle, G. Abba, and F. Plestan, "Asymptotically stable walking for biped robots: Analysis via systems with impulse effects," IEEE Trans. Autom. Control, vol. 46, no. 1, pp. 51-64, Jan. 2001.

[17] W. M. Haddad, V. Chellaboina, and S. G. Nersesov, Impulsive and Hybrid Dynamical Systems: Stability, Dissipativity, and Control. Princeton, NJ: Princeton Univ. Press, 2006.

[18] M. Heymann, F. Lin, G. Meyer, and S. Resmerita, "Analysis of Zeno behaviors in a class of hybrid systems," IEEE Trans. Autom. Control, vol. 50, no. 3, pp. 376-384, Mar. 2005.

[19] W. S. Howard and V. Kumar, "On the stability of grasped objects," IEEE Trans. Robot. Autom., vol. 12, no. 6, pp. 904-917, Dec. 1996.

[20] K. H. Johansson, M. Egerstedt, J. Lygeros, and S. Sastry, "On the regularization of Zeno hybrid automata," Syst. Control Lett., vol. 38, pp. $141-150,1999$.

[21] A. Lamperski and A. D. Ames, "Lyapunov-like conditions for the existence of Zeno behavior in hybrid and Lagrangian hybrid systems," in Proc. IEEE Conf. Decision and Control, 2007, pp. 115-120.

[22] A. Lamperski and A. D. Ames, "On the existence of Zeno behavior in hybrid systems with non-isolated Zeno equilibria," in Proc. IEEE Conf. Decision and Control, 2008, pp. 2776-2781.

[23] D. Liberzon, Switching in Systems and Control. Basel, Switzerland: Birkhauser, 2003

[24] D. Liberzon and M. Margaliot, "Lie-Algebraic stability conditions for nonlinear switched systems and differential inclusions," Syst. Control Lett., vol. 55, no. 1, pp. 8-16, 2006.

[25] C. Liu, Z. Zhen, and B. Brogliato, "Frictionless multiple impacts in multibody systems: Part I. Theoretical framework," Proc. Roy. Soc. A, vol. 464, no. 2100, pp. 3193-3211, 2008.

[26] A. M. Lyapunov, Stability of Motion. New York/London: Academic Press, 1966.

[27] J. Lygeros, K. H. Johansson, S. Simic, J. Zhang, and S. Sastry, "Dynamical properties of hybrid automata," IEEE Trans. Autom. Control, vol. 48 , pp. $2-17$, Feb. 2003 
[28] T. McGeer, "Passive walking with knees," in Proc. IEEE Int. Conf. Robotics and Automation, 1990, vol. 3, pp. 1640-1645.

[29] B. M. Miller and J. Bentsman, "Generalized solutions in systems with active unilateral constraints," Nonlin. Anal.: Hybrid Syst., vol. 1, pp. 510-526, 2007.

[30] R. M. Murray, Z. Li, and S. Sastry, A Mathematical Introduction to Robotic Manipulation. Boca Raton, FL: CRC Press, 1993.

[31] Y. Or and A. D. Ames, "Existence of periodic orbits with Zeno behavior in completed Lagrangian hybrid systems," in Hybrid Systems: Computation and Control, ser. Lecture Notes in Computer Science. New York: Springer-Verlag, 2009, pp. 291-305.

[32] Y. Or and E. Rimon, "On the hybrid dynamics of planar mechanisms supported by frictional contacts. I : Necessary conditions for stability," in Proc. IEEE Int. Conf. Robotics and Automation, 2008, pp. 1213-1218.

[33] Y. Or and E. Rimon, "On the hybrid dynamics of planar mechanisms supported by frictional contacts. II : Stability of two-contact rigid body postures," in Proc. IEEE Int. Conf. Robotics and Automation, 2008, pp. $1219-1224$.

[34] Y. Or and A. R. Teel, "Zeno stability of the set-valued bouncing ball," IEEE Trans. Autom. Control, vol. 56, no. 2, pp. 447-452, Feb. 2011.

[35] P. R. Pagilla and M. Tomizuka, "Contact transition control of nonlinear mechanical systems subject to a unilateral constraint," ASME J. Dynam. Syst., Measur., and Control, vol. 119, no. 4, pp. 749-759, 1997.

[36] F. Pfeiffer and C. Glocker, Multibody Dynamics With Unilateral Contacts. New York: Wiley, 1996.

[37] J. Pratt and G. A. Pratt, "Exploiting natural dynamics in the control of a planar bipedal walking robot," in Proc. 36th Annu. Allerton Conf. Communications, Control and Computing, 1998, pp. 739-748.

[38] R. Sanfelice, R. Goebel, and A. Teel, "Generalized solutions to hybrid dynamical systems," ESAIM: Control, Optimis., and Calculus of Variat, , vol. 14, pp. 699-724, 2008.

[39] R. Sanfelice, R. Goebel, and A. Teel, "Invariance principles for switching systems via hybrid systems techniques," IEEE Trans. Autom. Control, vol. 52, no. 12, pp. 2282-2297, Dec. 2007.

[40] S. Sastry, Nonlinear Systems: Analysis, Stability and Control. New York: Springer-Verlag, 1999.

[41] J. Shen and J.-S. Pang, "Linear complementarity systems: Zeno states," SIAM J. Control and Optimiz., vol. 44, no. 3, pp. 1040-1066, 2005.

[42] S. Simic, K. H. Johansson, J. Lygeros, and S. Sastry, "Structural stability of hybrid systems," in Proc. European Control Conf., 2001, pp. 3858-3863.

[43] M. W. Spong and F. Bullo, "Controlled symmetries and passive walking," IEEE Trans. Autom. Control, vol. 50, no. 7, pp. 1025-1031, Jul. 2005.

[44] A. van der Schaft and H. Schumacher, "An introduction to hybrid dynamical systems," in Lecture Notes in Control and Information Sciences 251. New York: Springer-Verlag, 2000.

[45] M. Vukobratovic and B. Borovac, "Zero moment point-thirty five years of its life," Int. J. Humanoid Robot., vol. 1, no. 1, pp. 157-173, 2004.

[46] E. R. Westervelt, J. W. Grizzle, C. Chevallereau, J. H. Choi, and B. Morris, Feedback Control of Dynamic Bipedal Robot Locomotion. Boca Raton, FL: CRC Press, 2007.
[47] H. Ye, A. N. Michel, and L. Hou, "Stability theory for hybrid dynamical systems," IEEE Trans. Autom. Control, vol. 43, no. 4, pp. 461-474, Apr. 1998.

[48] M. Zefran and V. Kumar, "Optimal control of systems with unilateral constraints," in Proc. IEEE Int. Conf. Robotics and Automation, 1995, pp. 2695-2700.

[49] J. Zhang, K. H. Johansson, J. Lygeros, and S. Sastry, "Dynamical systems revisited: Hybrid systems with Zeno executions," in Hybrid Systems: Computation and Control, ser. Lecture Notes in Computer Science. New York: Springer-Verlag, 2000, vol. 1790, pp. 451-464.

[50] J. Zhang, K. H. Johansson, J. Lygeros, and S. Sastry, "Zeno hybrid systems," Int. J. Robust Nonlin. Control, vol. 11, no. 2, pp. 435-451, 2001.

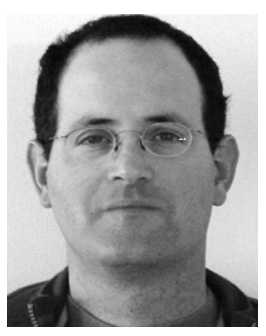

Yizhar Or received dual B.Sc. degrees (with highest honors) in mechanical engineering and education in computer science and the Ph.D. degree from the Technion-Israel Institute of Technology, Haifa, Israel, in 2001 and 2007, respectively.

$\mathrm{He}$ is currently a Lecturer at the Faculty of Mechanical Engineering and the Head of the Laboratory of Bio-Dynamics and Mechanics of Locomotion at the Technion.

Dr. Or received the Fulbright and Bikura Postdoctoral Fellowships and was a Postdoctoral Scholar at the Department of Control and Dynamical Systems, California Institute of Technology, Pasadena, from 2007 to 2009.

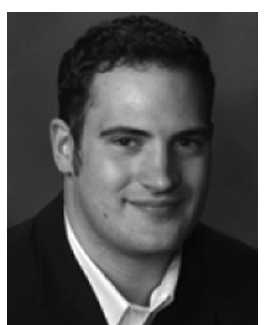

Aaron D. Ames (A'06) received the B.S. degree in mechanical engineering and the B.A. degree in mathematics from the University of St. Thomas, St. Paul, MN, in 2001, and the M.A. degree in mathematics and the Ph.D. degree in electrical engineering and computer sciences from the University of California Berkeley in 2006.

$\mathrm{He}$ is an Assistant Professor in Mechanical Engineering at Texas A\&M University, College Station. He served as a Postdoctoral Scholar in the Control and Dynamical System Department, California Institute of Technology, from 2006 to 2008 . His research interests center around dynamical, control, and hybrid systems, with a special emphasis on bipedal robots, behavior unique to hybrid systems, such as Zeno behavior, and the mathematical foundations of hybrid systems.

Dr. Ames received the 2005 Leon O. Chua Award from UC Berkeley for achievement in nonlinear science, the 2006 Bernard Friedman Memorial Prize in applied mathematics, and the NSF CAREER award for "Closing the Loop on Walking: From Hybrid Systems to Bipedal Robots to Prosthetic Devices and Back" in 2010. 